\title{
Polyspectra of Ordered Signals
}

\author{
Bernard Picinbono, Fellow, IEEE
}

\begin{abstract}
Polyspectra are related to Fourier transforms of moment or cumulant functions of any order of random signals. They play an important role in many problems of signal analysis and processing. However, there are only a few statistical models giving explicitly the expression of polyspectra. Ordered signals are signals for which the explicit expression of the moment functions requires that the time instants appearing in these moments are put in an increasing order. There are many examples of such signals, the best known being the random telegraph signal constructed from a Poisson process. Some of these examples are presented and analyzed. The origin of the ordering structure is related with the point that real time is an oriented variable making a difference between past and future. This especially appears in Markov processes. The calculation of polyspectra is difficult because ordering is not adapted to Fourier analysis. By an appropriate grouping of various terms, the explicit expression of spectral moment functions is obtained. It shows in particular that many ordered signals present a normal density on the normal manifolds of the frequency domain and another contribution on the stationary manifold that is explicitly calculated. The analysis of the structure of this expression allows us to discuss some relationships with normal distribution, central limit theorem, and time reversibility.
\end{abstract}

Index Terms - Central limit theorem, higher order statistics, normal distribution, signal analysis, spectral representation.

\section{INTRODUCTION}

$\mathbf{P}$ OLYSPECTRA play an especially important role in the methods of signal analysis and processing using statistics of an order higher than two (HOS). From the structure of polyspectra it is possible to deduce various properties of signals that do not appear when using the power spectrum only. For example, many quite different signals can have the same correlation function, or the same power spectrum, but they can be distinguished by using HOS. Furthermore, there are various methods of signal processing using polyspectra in order to solve problems that cannot be solved by only using secondorder statistics. There are many papers dealing with properties and applications of bi- or trispectrum and it is not possible here to give a significant list of these papers. Restricting our attention to signal analysis, it is worth pointing out the papers where the general structure or symmetry of polyspectra are studied [1]-[4]. However, the mathematical expression of polyspectra is in general difficult to write explicitly. This expression is well known in the case of strictly white signals, of normal signals, of Poisson processes (see [5, Chs. 7 and $8]$ ), and for linear processes or some nonlinear processes

Manuscript received September 7, 1997; revised March 26, 1999.

The author is with the Laboratoire des Signaux et Systèmes, Supélec, Plateau de Moulon, 91192 Gif sur Yvette, France (e-mail: picinbono@1ss.supelec. fr).

Communicated by C. Herley, Associate Editor for Estimation.

Publisher Item Identifier S 0018-9448(99)07311-3. characterized by a Volterra expansion (see [6, p. 39]). Many papers deal with polyspectra of nonnormal signals [7]-[9]. However, without any additional specification, only general structure can be used in this case, because nonnormality does not introduce any precise definition of the statistics of a signal. Therefore, if we require explicit analytic expressions of polyspectra, it is necessary to introduce statistical models of signals that can represent physical phenomena and have a structure leading to possible explicit calculations. This is one of the main purposes of this paper.

Many signals are related to physical phenomena or obtained by systems operating in real time. Therefore, the variable $t$ appearing in the signal $x(t)$ is not only a point on a straight line, but an oriented variable because time is going in one sense only. This is the basis of the questions related with causality. This also appears in HOS in which we study mean values of a signal at $n$ time instants $t_{1}, t_{2}, \cdots, t_{n}$. From a mathematical point of view these instants are arbitrary, and this is especially true for calculating the polyspectra. Indeed, this calculation requires a Fourier transformation in which the variables $t_{i}$ are independent variables going from $-\infty$ to $+\infty$. However, from a physical point view these instants must sometimes be put in an increasing order. This is especially the case for signals in which the future is constructed from the present and the past. Therefore, for such signals, here called ordered signals and very common in practical problems, calculation of polyspectra requires specific methods analyzed here.

The paper is organized as follows. In Section II, a short review of known concepts concerning moments and cumulants and their Fourier transforms is presented. Because of its importance in the analysis that follows, a specific emphasis is put on the stationary and normal manifolds in the frequency domain. There are surfaces characterizing the fact that a signal is stationary or normal, respectively. As relation to normality is an important issue, these manifolds will play an important role is the discussion that follows. Section III introduces the concept of ordered signals. These signals are characterized by the fact that the explicit expression of their moments or cumulants requires an ordered sequence of time instants. The simplest example of such signals is the famous random telegraph signal (RTS). Because of its simplicity it will be analyzed in detail. However, there are many other examples and some of them are presented and discussed. These signals frequently appear in physical applications because the ordering property is quite natural. For example, it appears with Markov processes for which past and future are independent, given the present. Then there is in the definition an ordering structure making a difference between past, present, and future. In Section IV, the principle of the calculation of the Fourier 
transforms of some ordered signals is presented. The difficulty comes from the fact that the explicit expression of the moment is known only for one permutation. Therefore, there exists $n$ ! different values of a moment of order $n$ and $n$ ! distinct domains for the calculation of the Fourier transform, which leads to a great deal of algebraic manipulations. Furthermore, the ordering property makes a strong difference between past and future, and, therefore, introduces in the time domain many unit step functions. As a consequence, the calculation of the Fourier transform leading to the polyspectra requires some specific and unusual properties of distributions. In Section $\mathrm{V}$, these calculations are used to obtain some polyspectra of ordered signals and to discuss their properties. One of the most important points to analyze is the value of the density on the normal manifolds and it is shown that for many ordered signals this density is the same as the one of a normal process (normal density). The last sections are devoted to the study of some consequences concerning central limit theorem and time reversibility. By central limit theorem we mean the fact that a nonnormal signal can become approximately normal after its filtering in a narrowband filter. On the other hand, reversibility means the fact that the statistical properties can remain invariant by time reversing. It is shown that these properties are strongly related to the value of the polyspectra on normal manifolds, which justifies the interest in analyzing their structures.

\section{REVIEW OF KNOWN RESUlTS}

Let $x(t)$ be a real random signal with zero mean value. The variable $t$ refers to the time and most of the following results are valid in continuous and discrete time. The $n$ th-order moment (or moment function) of $x(t)$ is defined by

$$
\begin{aligned}
m_{n}\left(\left\{t_{i}\right\}\right) & =m_{n}(\boldsymbol{t}) \\
& =m_{n}\left(t_{1}, t_{2}, \cdots, t_{n}\right) \\
& =E\left[x\left(t_{1}\right) x\left(t_{2}\right) \cdots x\left(t_{n}\right)\right] .
\end{aligned}
$$

If the stationarity assumption is introduced, this moment is a function of only $n-1$ variables. For $n=2$ we obtain the correlation function $x(t)$ written as $\gamma\left(t_{1}-t_{2}\right)$. When $x(t)$ is normal, or Gaussian, the moment (1) is zero for $n$ odd and for even values of $n$ takes the form

$$
m_{2 k}^{G}\left(\left\{t_{i}\right\}\right)=\sum_{\mathcal{P}} \prod \gamma\left(t_{i}-t_{j}\right)
$$

where the sum with respect to the normal (or Gaussian) partitions $\mathcal{P}$ contains $(2 k-1)$ !! terms generalizing in an obvious way the expression

$$
\begin{aligned}
m_{4}^{G}\left(\left\{t_{i}\right\}\right)= & \gamma\left(t_{1}-t_{2}\right) \gamma\left(t_{3}-t_{4}\right)+\gamma\left(t_{1}-t_{3}\right) \gamma\left(t_{2}-t_{4}\right) \\
& +\gamma\left(t_{1}-t_{4}\right) \gamma\left(t_{2}-t_{3}\right)
\end{aligned}
$$

valid for $2 k=4$. The letter $G$ refers to the Gaussian structure.

Instead of using the moments defined by (1), it is sometimes more interesting to use the cumulants functions introduced first in [10]. It is not appropriate to present here a discussion concerning respective advantages and disadvantages of moments and cumulants. Note simply that the cumulants of a normal signal are zero for $n>2$. Furthermore, there is the same information in moments or cumulants and the expression allowing the calculation of cumulants in terms of moments is given in [6, p. 19].

Polyspectra are related to the Fourier transforms (FT) of moments or cumulants. Let $M_{n}\left(\left\{f_{i}\right\}\right)=M_{n}(f)$ be the FT of (1) called here spectral moment function. If $x(t)$ is stationary this function can be written (see [5, p. 238]) as

$$
\begin{aligned}
M_{n}\left(\left\{f_{i}\right\}\right) & =M_{n}(f) \\
& =\Gamma_{n-1}\left(f_{1}, f_{2}, \cdots, f_{n-1}\right) \delta\left(f_{1}+f_{2}+\cdots+f_{n}\right)
\end{aligned}
$$

where $\delta(\cdot)$ is the Dirac distribution. This means that $M_{n}\left(\left\{f_{i}\right\}\right)=0$, except on the manifold of the space $f_{1} \times f_{2} \times \cdots \times f_{n}$ defined by $f_{1}+f_{2}+\cdots+f_{n}=0$ called stationary manifold. The function $\Gamma_{n-1}(\cdot)$ is called the polyspectrum of order $n-1$. The polyspectrum of order 1 is the classical power spectrum $\Gamma(f)$ of $x(t)$, FT of its correlation function. For practical applications, bi- and trispectra are the most often used polyspectra. The same procedure can be applied to the cumulants, which introduces the cumulant spectral functions and polyspectra.

For the discussion that follows it is especially interesting to consider the normal case. By Fourier transformation of (2) we obtain

$$
M_{2 k}^{G}\left(\left\{f_{i}\right\}\right)=\sum_{\mathcal{P}} \prod \Gamma\left(f_{i}\right) \delta\left(f_{i}+f_{j}\right)
$$

where the sum is calculated with respect to the normal partitions $\mathcal{P}$. For $2 k=4$ this gives

$$
\begin{aligned}
M_{4}^{G}\left(\left\{f_{i}\right\}\right)= & \Gamma\left(f_{1}\right) \Gamma\left(f_{3}\right) \delta\left(f_{1}+f_{2}\right) \delta\left(f_{3}+f_{4}\right) \\
& +\Gamma\left(f_{1}\right) \Gamma\left(f_{2}\right) \delta\left(f_{1}+f_{3}\right) \delta\left(f_{2}+f_{4}\right) \\
& +\Gamma\left(f_{1}\right) \Gamma\left(f_{2}\right) \delta\left(f_{1}+f_{4}\right) \delta\left(f_{2}+f_{3}\right) .
\end{aligned}
$$

This introduces the concept of normal manifolds and of normal density (see [5, p. 278]). It results from (5) that $M_{2 k}^{G}\left(\left\{f_{i}\right\}\right)$ is zero outside the manifolds defined by equations such that

$$
f_{1}+f_{2}=0 \quad f_{3}+f_{4}=0, \cdots, f_{2 k-1}+f_{2 k}=0 .
$$

There are $(2 k-1)$ !! such manifolds defined by the $(2 k-1)$ !! permutations of the $f_{i}$ 's giving distinct equations of this form. These manifolds are called in the following normal manifolds and are clearly submanifolds of the stationary manifold defined after (4). Furthermore, the density on these normal manifolds is not arbitrary, but appears as a product of spectral densities of the signal and this structure is called a normal density. These properties can clearly be expressed in terms of polyspectra by applying (4) to (5). For example, the moment trispectrum of a normal signal is

$$
\begin{aligned}
\Gamma_{3}^{G}\left(f_{1}, f_{2}, f_{3}\right)= & \Gamma\left(f_{1}\right) \Gamma\left(f_{3}\right) \delta\left(f_{1}+f_{2}\right) \\
& +\Gamma\left(f_{1}\right) \Gamma\left(f_{2}\right) \delta\left(f_{1}+f_{3}\right) \\
& +\Gamma\left(f_{1}\right) \Gamma\left(f_{2}\right) \delta\left(f_{2}+f_{3}\right) .
\end{aligned}
$$

The first normal manifold for the trispectrum is then defined by $f_{1}+f_{2}=0$. In the space $f_{1} \times f_{2} \times f_{3}$ it is the plane defined by two straight lines: the $f_{3}$ axis defined by $f_{1}=f_{2}=0$ and 
the bisectrix of the $f_{1}, f_{2}$ axes defined by $f_{1}=-f_{2}, f_{3}=0$. On this plane the normal density is $\Gamma\left(f_{1}\right) \Gamma\left(f_{3}\right)$. The other two normal manifolds are defined by $f_{1}+f_{3}=0$ and $f_{2}+f_{3}=0$, respectively.

Conversely, if the function $M_{n}\left(\left\{f_{i}\right\}\right)$ defined by (4) has the normal structure characterized by (5) for any value of $k$, the moment (1) has the form (2). As the sequence of all the moments defines the probability distribution, a sequence of normal moments means that the signal is normal. As a result, a nonnormal process with a zero mean value has a spectral moment function that does not satisfy at least one of the following properties: 1) $\left.M_{2 k+1}\left(\left\{f_{i}\right\}\right)=0,2\right) M_{2 k}\left(\left\{f_{i}\right\}\right)=0$ outside the normal manifolds, and 3$)$ the density on the normal manifolds is not normal. For example, spherically invariant processes (see [5, p. 299]) have spectral moment functions (4) equal to zero for $n=2 k+1$ and outside the normal manifolds for $n=2 k$, but the density on the normal manifolds is not normal. In other words, the spectral moment functions are the same as those of normal signals, except the value of the density on the normal manifolds.

The structure of the density on the normal manifolds is important for various questions as ergodicity [11], central limit theorem for signals, and time reversibility analyzed in the following for ordered signals.

Let us consider the cumulant polyspectra noted $\Gamma_{c, n}\left(f_{1}, f_{2}, \cdots, f_{n}\right)$. They are defined exactly by the same procedure in which the moment function appearing in (1) is replaced by the cumulant function. These polyspectra are especially interesting in two cases. The first appears if the signal is normal. Indeed, the only nonzero polyspectrum of a normal signal is the power spectrum. This is an interesting characteristics or normality. The second case corresponds to the discrete-time strictly white noise. This means that $x[k]$ is a sequence of independent and identically distributed (i.i.d.) random variables with cumulants $c_{n}$. It results from the basic properties of cumulants that the cumulant polyspectrum appearing in (4) is now $\Gamma_{c, n-1}=c_{n}$. The same expression for the moments instead of cumulants is, of course, much more complex to write explicitly. However, for the following discussion it is interesting to study more carefully the relation between moment and cumulant polyspectra of discrete-time white noise. Let us first consider the case of the trispectrum. The moment trispectrum of white noise is

$$
\begin{aligned}
& \Gamma_{3}\left(f_{1}, f_{2}, f_{3}\right) \\
& \quad=c_{2}^{2}\left[\delta\left(f_{1}+f_{2}\right)+\delta\left(f_{1}+f_{3}\right)+\delta\left(f_{2}+f_{3}\right)\right]+c_{4}
\end{aligned}
$$

while the cumulant trispectrum is simply $c_{4}$. This shows that, contrary to the cumulant trispectrum, the moment trispectrum is not bounded in the space $f_{1} \times f_{2} \times f_{3}$ because of the presence of the delta distributions appearing in this equation. This is often considered as the most important disadvantage of moment trispectrum with respect to cumulant trispectrum, and this appears not only for the trispectrum but for any polyspectrum. Furthermore, (9) shows that the spectral moment function $M_{4}\left(\left\{f_{i}\right\}\right)$ appearing in (4) is distributed on the normal manifolds with a normal density and also on the stationary manifold with the density $c_{4}$. These points, shown for the trispectrum, are general for polyspectra of any order and lead to a geometrical interpretation of the relation between moments and cumulants. For this, consider the spectral moment function $M_{6}\left(\left\{f_{i}\right\}\right)$ of white noise. This function is zero outside the stationary manifold but is also distributed on some of its submanifolds. There are three kinds of such manifolds. The first ones are the normal manifolds defined by equations such that $f_{1}+f_{2}=0, f_{3}+f_{4}=0$, $f_{5}+f_{6}=0$, and all their distinct permutations. The density on these manifolds is $m_{2}^{3}$, where $m_{n}$ is the $n$ th-order moment of the i.i.d. random variables defining the white noise. The second submanifolds are on the type $f_{1}+f_{2}+f_{3}=0, f_{4}+f_{5}+f_{6}=0$ and the density is now $m_{3}^{2}$. Finally, there are the manifolds $f_{1}+f_{2}+f_{3}+f_{4}=0, f_{5}+f_{6}=0$, and all their distinct permutations, introducing the density $m_{2} m_{4}$. In order to obtain a bounded spectral cumulant it suffices to subtract from the spectral moment all these terms and making this operation gives the relation giving moments in terms of cumulants. The same discussion can be presented for the continuoustime white noise by using known results concerning general properties of processes with independent increments.

The fact that the cumulant polyspectra are bounded is not specific to white noise and is true for many other signals. This is especially the case for linear processes [6], which means signals obtained at the output of a linear filter driven by a white noise. The cumulant function $c\left(t_{1}, t_{2}, \cdots, t_{n}\right)$ in the continuous-time case is given by

$$
\begin{aligned}
c_{n}\left(t_{1}, t_{2}, \cdots, t_{n}\right) \\
\quad=c_{n} \int h\left(t_{1}-\theta\right) h\left(t_{2}-\theta\right) \cdots h\left(t_{n}-\theta\right) d \theta
\end{aligned}
$$

where $c_{n}$ characterizes the driving white noise and $h(t)$ is the impulse response of the filter. The cumulant polyspectra are, therefore,

$$
\begin{aligned}
\Gamma_{c, n-1} & \left(f_{1}, f_{2}, \cdots, f_{n-1}\right) \\
= & c_{n} H\left(f_{1}\right) H\left(f_{2}\right) \cdots H\left(f_{n-1}\right) \\
& \times H\left(-f_{1}-f_{2}-\cdots-f_{n-1}\right)
\end{aligned}
$$

where $H(f)$ is the frequency response of the filter. When this filter is real we can use the Hermitian symmetry $H(-f)=$ $H^{*}(f)$ to simplify this expression. It shows obviously that if $H(f)$ is bounded the cumulant polyspectra are also bounded. However, it must be noted that this property is not valid for every signal and there are many counterexamples. The simplest is certainly that of spherically invariant processes introduced above. One can show, for example (see [5, p. 280]), that their cumulant trispectrum can be written as $k \Gamma_{3}^{G}\left(f_{1}, f_{2}, f_{3}\right)$, where $k=0$ in the normal case. It is obvious from (8) that this spectrum is not bounded in the space $f_{1} \times f_{2} \times f_{3}$.

Furthermore, note that (10) introduces a necessary condition in the time domain that must satisfy a signal to be a linear process. By integration of (10) with respect to some time instants we obtain easily if $c_{m} \neq 0$

$$
\begin{aligned}
\int \cdots \int c_{n}\left(t_{1}, t_{2},\right. & \left.\cdots, t_{m}, t_{m+1}, t_{m+2}, \cdots, t_{n}\right) \\
& \times d t_{m+1} d t_{m+2} \cdots d t_{n} \\
& =\frac{c_{n}[H(0)]^{n-m}}{c_{m}} c_{m}\left(t_{1}, t_{2}, \cdots, t_{m}\right) .
\end{aligned}
$$


In this expression, it is useful to note that $H(0)$ is related to the power spectrum by the relation $\Gamma(0)=c_{2}|H(0)|^{2}$.

Linear processes are the most used in practice and (11) shows that their polyspectra possess a factorization property. Note, however, that a factorization such as (11) does not imply that the signal is a linear process. For this, (11) must be satisfied for any $n$ and, furthermore, the coefficients appearing in this factorization must satisfy various conditions ensuring that a set of numbers $c_{n}$ is a set of cumulants. These conditions appear in the famous "moment problem" of probability theory.

There is a point important to note concerning the HOS of random signals. Any second-order signal can be considered as generated by a linear filter driven by white noise. This only means that the signal and its linear model have the same correlation function. However, these two signals have no reason to be identical. This is no longer true for HOS and, for example, if (12) is not satisfied, there is no linear filter and white noise giving an output with the same statistics.

Note finally that when no a priori knowledge of a signal is introduced the calculation of the cumulant functions requires the use of the very complex expression giving these functions in terms of moments function and this is the reason why most of the calculations that follow are realized with moments, and cumulants are only sometimes introduced.

\section{ORDERED SIGNALS}

\section{A. Introduction and Definition}

The function $m_{n}\left(\left\{t_{i}\right\}\right)$ defined by (1) is symmetric with respect to the variables $t_{i}$. This means that it is invariant under the $n$ ! permutations of these time instants. Let us call $\theta_{i}$ the time instants deduced from the $t_{i}$ 's by the permutation ensuring that $\theta_{i} \leq \theta_{i+1}$. We shall say that the $\theta_{i}$ 's constitute the ordered permutation of the $t_{i}$ 's. It is clear that $m_{n}\left(\left\{t_{i}\right\}\right)$ is known as soon as $m_{n}\left(\left\{\theta_{i}\right\}\right)$ is known.

There are signals for which $m_{n}\left(\left\{t_{i}\right\}\right)$ is explicitly defined for any value of the $t_{i}$ 's. This is, for example, the case of the normal signals, according to (3). However, this is not always the case.

Ordered signals are signals for which only $m_{n}\left(\left\{\theta_{i}\right\}\right)$ is known, which means that the explicit expression of $m_{n}\left(\left\{t_{i}\right\}\right)$ is known only for the ordered permutation of the $t_{i}$ 's. Various examples of such signals will be presented hereafter. It is worth pointing out that for $n=2$ this ordering property is described by the absolute value. For example, the correlation function $\exp \left[\left(t_{1}-t_{2}\right)^{2}\right]$ is defined for any set of $t_{i}$ 's. On the other hand, the correlation function $\exp \left[\left|t_{1}-t_{2}\right|\right]$ takes two distinct explicit expressions according to the sign of $t_{1}-t_{2}$.

It is well known that the symmetry property of $m_{n}\left(\left\{t_{i}\right\}\right)$ has its counterpart in the Fourier domain. The obvious consequence is that the spectral moment function $M_{n}\left(\left\{f_{i}\right\}\right)$ appearing in (4) is symmetric with respect to the $f_{i}$ 's. This implies that this property is also valid for the polyspectrum $\Gamma_{n}\left(\left\{f_{i}\right\}\right)$ appearing in (4). But, because of the delta term, the polyspectrum is invariant when replacing any frequency by $f_{n}=-\left(f_{1}+f_{2}+\cdots+f_{n-1}\right)$. This was used in many papers discussing the minimum domain of definition of the polyspectrum. On the other hand, the ordered property has no direct consequence on polyspectra because the structure of such polyspectra is a consequence of their calculation, which is the purpose of this paper.

There is a large class of signals for which the explicit expression of the moment function (1) requires that the distinct times instant $t_{i}$ be classed in a given order. This order defines a specific permutation $\left(\left\{\theta_{i}\right\}\right)$ of the time instants $\left(\left\{t_{i}\right\}\right)$ and the expression of $m_{n}\left(\left\{t_{i}\right\}\right)$ is given in terms of the $\theta_{i}$ 's. This property is the origin of the expression of ordered signals.

It is worth pointing out that this is not in contradiction with the symmetry property of the moments meaning that $m_{n}\left(\left\{t_{i}\right\}\right)$ is invariant under any permutation of the $t_{i}$ 's. Indeed, any permutation of a given set of $n$ instants $t_{i}$ does not change the unique set of instants $\theta_{i}$ obtained by an ordering of the $t_{i}$ 's

We shall now present the simplest examples of ordered signals and afterwards discuss the origin of the ordering property.

\section{B. Random Telegraph Signal (RTS)}

It is a signal which only takes the values of \pm 1 with the same probabilities, the changes of signs arising at the time instants $p_{i}$ of a stationary Poisson point process of density $\lambda$. Its correlation function is

$$
m_{2}\left(t_{1}, t_{2}\right)=\gamma\left(t_{1}-t_{2}\right)=\exp \left(-2 \lambda\left|t_{1}-t_{2}\right|\right) .
$$

The moments (1) are zero for odd values of $n$ and for even values one obtains (see [5, p. 334])

$$
m_{2 k}\left(\left\{t_{i}\right\}\right)=\gamma\left(\theta_{2}-\theta_{1}\right) \gamma\left(\theta_{4}-\theta_{3}\right) \cdots \gamma\left(\theta_{2 k}-\theta_{2 k-1}\right)
$$

where the time instants $\theta_{i}$ are obtained by the unique permutation of the $2 k$ distinct $t_{i}$ 's such that $\theta_{i}<\theta_{i+1}$. This is the simplest example of ordered signal discussed hereafter. It is interesting to compare this expression with (3) valid for $k=2$ and normal signals. In this case, there are three terms due to the three normal partitions, while (13) exhibits only one term due to the only one ordered permutation. Note especially that for this signal it is obviously much easier to work with moments than with cumulants. Indeed, using the classical expression giving cumulants in terms of moments leads to a very complicated mathematical expression. However, we see immediately that if the moments are simple to write explicitly the calculation of polyspectra remains complicated because of the ordered structure that is not appropriate for a simple Fourier transformation.

\section{Random Jump Signals}

In the studies concerning abrupt changes of the state of systems, one can describe the situation by the following signal. The starting point is once again a Poisson process of density $\lambda$. At each time instant $p_{i}$ of the process we choose a random variable $V_{i}$ with a zero mean value. We assume that all these random variables are i.i.d. and also independent of the Poisson process. The signal $x(t)$ is defined as equal to $V_{i}$ for $p_{i} \leq t<p_{i+1}$. This is, therefore, a constant signal with random jumps at the points of the Poisson process. Hence, 
the statistics of the signal are completely described by the moments $m_{n}$ of the random variables $V_{i}$ and by the density $\lambda$ of the Poisson process. As before, it is assumed that $m_{0}=0$. Let us indicate the principle of the calculations of the moments functions.

Let $\theta_{1}$ and $\theta_{2}$ be two distinct arbitrary and ordered $\left(\theta_{1}<\theta_{2}\right)$ time instants. If there is at least one point $p_{i}$ of the process in the interval $\left(\theta_{1}, \theta_{2}\right), x\left(\theta_{1}\right)$, and $x\left(\theta_{2}\right)$ are independent and $E\left[x\left(\theta_{1}\right) x\left(\theta_{2}\right)\right]=0$. If there is no point in this interval, an event with probability $\exp \left[-\lambda\left(\theta_{2}-\theta_{1}\right)\right], E\left[x\left(\theta_{1}\right) x\left(\theta_{2}\right)\right]=m_{2}$. Thus for any $t_{1}$ and $t_{2}$, the correlation function of $x(t)$ is

$$
m_{2}\left(t_{1}, t_{2}\right)=\gamma\left(t_{1}-t_{2}\right)=m_{2} \exp \left[-\lambda\left|t_{2}-t_{1}\right|\right] .
$$

Let us now calculate the third-order moment defined by (1). The reasoning is the same. Let $\theta_{i}$ 's be the time instants obtained by the ordered permutation of the $t_{i}$ 's. Because of the assumption of zero mean value $E\left[x\left(t_{1}\right) x\left(t_{2}\right) x\left(t_{3}\right)\right]=0$ as soon as there is at least one point $p_{i}$ between $\theta_{1}$ and $\theta_{3}$. This yields

$$
m_{3}\left(t_{1}, t_{2}, t_{3}\right)=m_{3} \exp \left[-\lambda\left(\theta_{3}-\theta_{1}\right)\right]
$$

and the interesting point is that this expression does not depend on the intermediary time $\theta_{2}$.

Consider now the fourth-order moment (1). The $\theta_{i}$ 's define still the ordered permutation of the distinct $t_{i}$ 's. Let $X$ be the random variable

$$
X=x\left(\theta_{1}\right) x\left(\theta_{2}\right) x\left(\theta_{3}\right) x\left(\theta_{4}\right)=x\left(t_{1}\right) x\left(t_{2}\right) x\left(t_{3}\right) x\left(t_{4}\right) .
$$

Because of the assumption of independence and zero mean value, $E(X) \neq 0$ only if there is no point of the Poisson process in the intervals $\left(\theta_{1}, \theta_{2}\right)$ and $\left(\theta_{3}, \theta_{4}\right)$. Moreover, if there is also no point in $\left(\theta_{2}, \theta_{3}\right)$, then $E(X)=m_{4}$, and if there is at least one point in this interval, we have $E(X)=m_{2}^{2}$. Combining these results we deduce that

$$
\begin{aligned}
m_{4}\left(\left\{t_{i}\right\}\right)= & m_{2}^{2}\left[1-e^{-\lambda\left(\theta_{3}-\theta_{2}\right)}\right]\left[e^{-\lambda\left(\theta_{2}-\theta_{1}\right)} e^{-\lambda\left(\theta_{4}-\theta_{3}\right)}\right] \\
& +m_{4} e^{-\lambda\left(\theta_{4}-\theta_{1}\right)}
\end{aligned}
$$

This can be expressed in the form

$$
m_{4}\left(\left\{t_{i}\right\}\right)=\gamma\left(\theta_{2}-\theta_{1}\right) \gamma\left(\theta_{4}-\theta_{3}\right)+\left(m_{4}-m_{2}^{2}\right) e^{-\lambda\left(\theta_{4}-\theta_{1}\right)}
$$

It is worth pointing out that $m_{4}-m_{2}^{2}$ is the variance of the random variable $V_{i}^{2}$ and if $m_{4}=m_{2}^{2}$, then $V_{i}^{2}$ is no longer random. This implies that the random variables $V_{i}$ can only take the values $\pm \sqrt{m_{2}}$, and, as the mean is zero, these two values have the same probability. In this case, the trajectories of the signal $x(t)$ are similar to those of the RTS, with the big difference that the changes of the sign are random instead of deterministic. That is the reason we obtain the term $\lambda$ instead of $2 \lambda$ in the correlation function (14). Furthermore, (17) is a sum of two terms. The first one is similar to (13) valid for the RTS while the latter has the same structure as (15).

The same principles can be used for the calculations of the moments (1) for any value of $n$. However, the expressions become more and more tedious to write explicitly.
It is worth noting that the RTS and the random jump signal have the same exponential correlation function, even though they are quite different signals. The RTS only takes two values while the random jump signal takes the possible values of the random variable $V$ which is arbitrary, provided that its moments do exist. First this means that all the methods using only the correlation function will give the same result. This is especially the case in the linear prediction, and therefore the prediction innovation is the same. As a consequence they have the same linear representation. Second, this shows the interest of HOS, because these two signals can be distinguished by third-order moments, or by their trispectrum.

Finally, let us show that the random-jump signal cannot be a linear process. Indeed, applying (12) and noting that for $n=2$ and $n=3$ moments and cumulants are equal yield

$$
\int m_{3}\left(t_{1}, t_{2}, t_{3}\right) d t_{3}=\alpha \gamma\left(t_{1}-t_{2}\right)
$$

where $\alpha$ is a constant and $\gamma(\tau)$ is the correlation function. This is a necessary condition in terms of moments to obtain a linear process. By choosing $t_{2}=0$ and $t_{1}>0$ we obtain that the integral appearing in (18) is proportional to $\left[t_{1}+2 / \lambda\right] \exp \left(-\lambda t_{1}\right)$, which means that (18) is not satisfied.

This model can be generalized by replacing the random variable $V_{i}$ by random signals $v_{i}(t)$ with the same assumptions of independence. Assuming that all these signals have the same statistical properties and reasoning the same way as previously yields the correlation function

$$
\begin{aligned}
m_{2}\left(t_{1}, t_{2}\right) & =\gamma\left(t_{1}-t_{2}\right) \\
& =\exp \left[-\lambda\left(\left|t_{2}-t_{1}\right|\right)\right] c\left(t_{2}-t_{1}\right)
\end{aligned}
$$

where $c\left(t_{2}-t_{1}\right)$ is the correlation function common to all the signals of the model. Similarly, the third-order moment takes the form

$$
m_{3}\left(t_{1}, t_{2}, t_{3}\right)=\mu_{3}\left(t_{1}, t_{2}, t_{3}\right) \exp \left[-\lambda\left(\theta_{3}-\theta_{1}\right)\right]
$$

where $\mu_{3}\left(t_{1}, t_{2}, t_{3}\right)$ is the third-order moment function (1) common to all the signals $v_{i}(t)$ of the model. Finally, the fourth-order moment is

$$
\begin{aligned}
m_{4}\left(\left\{t_{i}\right\}\right)= & \gamma\left(\theta_{2}-\theta_{1}\right) \gamma\left(\theta_{4}-\theta_{3}\right) \\
& +\left[\mu_{4}\left(\left\{t_{i}\right\}\right)-c\left(\theta_{2}-\theta_{1}\right) c\left(\theta_{4}-\theta_{3}\right)\right] e^{-\lambda\left(\theta_{4}-\theta_{1}\right)}
\end{aligned}
$$

where $\mu_{4}(\cdot)$ is the moment (1) of the signals $v_{i}(t)$. All these examples of fourth-order moments exhibit two common properties: the first is that the explicit expression of $m_{4}\left(\left\{t_{i}\right\}\right)$ requires an ordering of the time instants $t_{i}$; the latter is that, even though the expressions are different, the correlation function $\gamma(\cdot)$ of the signal appears in the form $\gamma\left(\theta_{2}-\theta_{1}\right) \gamma\left(\theta_{4}-\theta_{3}\right)$.

\section{Switching Signals}

It is a direct extension of the RTS and can be introduced as follows. Let $u(t)$ and $v(t)$ be two independent random signals with the same probability distributions and zero mean values. Starting once again from a stationary Poisson process 
of density $\lambda$, let $x(t)$ be the signal switching from $u(t)$ to $v(t)$ or from $v(t)$ to $u(t)$ at each point $p_{i}$ of the Poisson process.

Let $c(\cdot)$ be the correlation function common to $u(t)$ and $v(t)$. Consider two arbitrary distinct time instants $\left(t_{1}, t_{2}\right)$, and let $\left(\theta_{1}, \theta_{2}\right)$ be their ordered permutation. If there is an odd number of points $p_{i}$ in the interval $\left(\theta_{1}, \theta_{2}\right), E\left[x\left(\theta_{1}\right) x\left(\theta_{2}\right)\right]=0$ because $u(t)$ and $v(t)$ are independent and with a zero mean value. On the other hand, if there is an even number of points $p_{i}, x\left(\theta_{1}\right)$ and $x\left(\theta_{2}\right)$ are values of the same function, either $u(t)$ or $v(t)$. The probability of obtaining an even number of points in a Poisson process is $\exp (-m) \cosh m$ with $m=\lambda\left(\theta_{2}-\theta_{1}\right)$. As a result we obtain

$$
\begin{aligned}
m_{2}\left(t_{1}, t_{2}\right)= & \gamma\left(t_{1}-t_{2}\right) \\
= & \exp \left[-\lambda\left(\left|t_{2}-t_{1}\right|\right)\right] \\
& \times \cosh \left[\lambda\left(\left|t_{2}-t_{1}\right|\right)\right] c\left(t_{2}-t_{1}\right)
\end{aligned}
$$

which is similar to (19).

Consider now four distinct times $t_{i}$ and let $\theta_{i}$ be their ordered permutation. Let $N_{1}, N_{2}$, and $N_{3}$ be the random number of points $p_{i}$ in the time interval $\left[\theta_{1}, \theta_{2}\right],\left[\theta_{2}, \theta_{3}\right]$, and $\left[\theta_{3}, \theta_{4}\right]$, respectively. Finally, let $X$ be the random variable $x\left(\theta_{1}\right) x\left(\theta_{2}\right) x\left(\theta_{3}\right) x\left(\theta_{4}\right)$. In order to obtain $E(X) \neq 0$ it is necessary that the numbers $N_{1}$ and $N_{3}$ be even. If $N_{2}$ is even, the four values $x\left(\theta_{i}\right)$ are taken from the same signal, either $u(t)$ or $v(t)$, and $E(X)=\mu_{4}\left(\left\{\theta_{i}\right\}\right)$. On the other hand, if $N_{2}$ is odd, $E(X)=c\left(\theta_{2}-\theta_{1}\right) c\left(\theta_{4}-\theta_{3}\right)$. Combining these results yields

$$
\begin{aligned}
m_{4}\left(\left\{t_{i}\right\}\right)= & p_{+}\left(\theta_{2}-\theta_{1}\right) p_{+}\left(\theta_{4}-\theta_{3}\right)\left[p_{-}\left(\theta_{3}-\theta_{2}\right) c\left(\theta_{2}-\theta_{1}\right)\right. \\
& \left.\times c\left(\theta_{4}-\theta_{3}\right)+p_{+}\left(\theta_{3}-\theta_{2}\right) \mu_{4}\left(\left\{t_{i}\right\}\right)\right]
\end{aligned}
$$

where

$$
p_{+}\left(\theta_{i}-\theta_{j}\right) \text { and } p_{-}\left(\theta_{i}-\theta_{j}\right)
$$

are equal to

$$
\exp (-m) \cosh m \text { and } \exp (-m) \sinh m
$$

respectively, with $m=\lambda\left(\theta_{i}-\theta_{j}\right)$. This expression is similar to (17), but more complicated. However, it is a sum of two terms, in which a factor such as $a\left(\theta_{2}-\theta_{1}\right) b\left(\theta_{3}-\theta_{2}\right) a\left(\theta_{4}-\theta_{3}\right)$ appears. In the first term we have $a(t)=p_{+}(t) c(t)$ and $b(t)=p_{-}(t)$, while in the second term we have $a(t)=b(t)=p_{+}(t)$.

\section{E. Origin of the Ordering Property and Extensions}

The RTS is one of the simplest example of continuous-time Markov process and the ordering property appears for many other Markov processes. Furthermore, the point common to all the signals presented before is that they are obtained from a Poisson process, which means a process with independent increments. It is precisely this property that introduces the ordering procedure for the calculations of higher order moments. Indeed, the time instants must be taken in an increasing order to use the independence property of successive increments. As a result it is tempting to verify whether or not other ordered signals can be obtained from other processes with independent increments. In the continuous-time cases, the degree of freedom is not very large because the only other kind of such process is the Brownian motion, introducing independent increments with normal distribution.
In this perspective there is a well-known signal that can be considered. It is the phase-noise signal introduced in the studies of frequency or phase stability of oscillators and also in the problems of coherence of laser light [12]. It is a complex signal defined by

$$
z(t)=\exp \left[j \omega_{0} t+\int_{0}^{t} d w(\theta)+\Phi\right]
$$

where $w(t)$ is a Brownian motion of with diffusion constant $c$ and $\Phi$ a random phase uniformly distributed between 0 and $2 \pi$. This assumption ensures the stationarity of the signal and also the circularity property [13]. As a consequence, the only nonzero second-order moment is the correlation function $\gamma(\tau)=E\left[z(t) z^{*}(t-\tau)\right]$ which is

$$
\gamma(\tau)=\exp \left(j \omega_{0} \tau\right) E\left\{\exp \left[j \int_{t-\tau}^{t} d w(\theta)\right]\right\}
$$

The integral appearing in this equation is a normal random variable with variance $c \tau$, which implies that the correlation function of the signal is $\gamma(\tau)=\exp \left(j \omega_{0} \tau\right) \exp (-c|\tau|)$. By using the same definition of the $\theta_{i}$ 's as previously and also again the property of independent increments we find that

$$
E\left[z\left(\theta_{2}\right) z^{*}\left(\theta_{1}\right) z\left(\theta_{4}\right) z^{*}\left(\theta_{3}\right)\right]=\gamma\left(\theta_{2}-\theta_{1}\right) \gamma\left(\theta_{4}-\theta_{3}\right)
$$

which is similar to (13) and introduces an ordering structure. Because of the circularity property all the other fourth-order moments are zero. However, there is a strong difference with the case of the RTS. Indeed, when permuting the time instants in (26) we must also permute the position of the complex conjugate, which introduces a strong difference with the real case and various changes in the calculations that follow. That is why we shall restrict the following analysis to real signals.

On the other hand, many of the previous results can be extended to the case of compound Poisson processes. These processes appear in many physical situations, and especially in statistical optics (see [5, p. 345]). A compound Poisson process is a Poisson process with a random density. This means that in all the previous calculations of moments it is necessary to take an expectation with respect to the positive random variable $\lambda$. It is even possible to assume that $\lambda$ is replaced by a positive random process $\lambda(t)$, but this case is not analyzed in this paper.

Finally, note that the transposition to the discrete-time does not introduce any difficulty. Any sequence of i.i.d. random variables defines a random-walk process which has independent increments. From this process various ordered signals can easily be defined. For the calculations of polyspectra the difference is that integrals are replaced by series and the frequency domain is now limited to a finite interval, say $-1 / 2,+1 / 2$.

\section{Fourier TRANSFORMS OF SOME ORdERED FunCtions}

\section{A. Introduction and Notation}

The moment of order $2 k$ of the RTS is given by (13) and this is a first example of ordered function. This means a function such that its value for any $t_{i}$ is given by an expression where the $t_{i}$ 's must be replaced by the $\theta_{i}$ 's obtained by the 
permutation of the $t_{i}$ 's such that $\theta_{i}<\theta_{i+1}$. For the discussion that follows we shall use more general ordered functions defined by

$$
\begin{aligned}
s(\boldsymbol{t}) & =s\left(\theta_{1}, \theta_{2}, \cdots, \theta_{n}\right) \\
& =s_{1}\left(\theta_{2}-\theta_{1}\right) s_{2}\left(\theta_{3}-\theta_{2}\right) \cdots s_{n-1}\left(\theta_{n}-\theta_{n-1}\right) .
\end{aligned}
$$

The moment function of the RTS is obtained by taking $n=2 k$, $s_{1}=s_{3}=\cdots=s_{2 k-1}=\gamma$, and $s_{2 k}=1$. Similarly, (15) can be expressed in this form by using the relation

$$
\exp \left[-\lambda\left(\theta_{3}-\theta_{1}\right)\right]=\exp \left[-\lambda\left(\theta_{3}-\theta_{2}\right)\right] \exp \left[-\lambda\left(\theta_{2}-\theta_{1}\right)\right]
$$

Finally, the function $s(\boldsymbol{t})=1$ is obtained by replacing in (27) all the functions $s_{i}(\cdot)$ by 1 . Now the problem we want to solve is to calculate the FT $S(\boldsymbol{f})$ defined by

$$
S(\boldsymbol{f})=\int s(\boldsymbol{t}) \exp \left(-2 \pi j \boldsymbol{f}^{T} \boldsymbol{t}\right) d \boldsymbol{t}
$$

where $s(\boldsymbol{t})$ is an ordered function defined by (27).

\section{B. Principle of the Calculation}

Let denote $P$ the ordering permutation, or the permutation that transforms the $t_{i}$ 's into the $\theta_{i}$ 's, defined by

$$
P\left[t_{1}, t_{2}, \cdots, t_{n}\right]=\theta_{1}, \theta_{2}, \cdots, \theta_{n}, \quad \theta_{i}<\theta_{i+1} .
$$

Applying this permutation to the frequencies $f_{i}$ yields the frequencies $\mu_{i}$ defined by

$$
P\left[f_{1}, f_{2}, \cdots, f_{n}\right]=\mu_{1}, \mu_{2}, \cdots, \mu_{n} .
$$

It is obvious that the scalar product appearing in (28) remains the same in this permutation, or that

$$
f^{T} \boldsymbol{t}=\sum f_{i} t_{i}=\sum \mu_{i} \theta_{i} .
$$

To each permutation $P$ we can associate a contribution to the FT defined by

$$
\begin{aligned}
S_{P}\left(\left\{\mu_{i}\right\}\right)= & \iint \cdots \int_{D(P)} s\left(\left\{\theta_{i}\right\}\right) \exp \left[-2 \pi j \sum \mu_{i} \theta_{i}\right] \\
& \times d \theta_{1} d \theta_{2} \cdots d \theta_{n}
\end{aligned}
$$

where $D(P)$ is the domain defined by the relation $\theta_{1}<\theta_{2}<$ $\cdots<\theta_{n}$. Finally, the FT of $s(\boldsymbol{t})$ is

$$
S(\boldsymbol{f})=\sum_{P} S_{P}\left(\left\{\mu_{i}\right\}\right)
$$

where the sum is extended to all the $n$ ! permutations of the frequencies $f_{i}$.

It remains to calculate the functions $S_{P}\left(\left\{\mu_{i}\right\}\right)$. This quantity is defined by

$$
\begin{aligned}
S_{P}\left(\left\{\mu_{i}\right\}\right)= & \int_{-\infty}^{+\infty} d \theta_{1} \int_{\theta_{1}}^{+\infty} d \theta_{2} \cdots \int_{\theta_{n-1}}^{+\infty} d \theta_{n} s\left(\left\{\theta_{i}\right\}\right) \\
& \times \exp \left[-2 \pi j \sum \mu_{i} \theta_{i}\right]
\end{aligned}
$$

where $s(\cdot)$ is defined by (27). The integral with respect to $\theta_{n}$ yields

$$
I_{n}=\exp \left(-2 \pi j \mu_{n} \theta_{n-1}\right) S_{n-1,+}\left(\mu_{n}\right)
$$

where $S_{n-1,+}(\mu)$ is defined by

$$
S_{n-1,+}(\mu)=\int_{0}^{+\infty} s_{n-1}(\theta) \exp (-2 \pi j \mu \theta) d \theta
$$

or is the one-sided FT of $s_{n-1}(t)$. Repeating the same procedure for the integration with respect to the other variables $\theta_{i}$ yields

$$
\begin{aligned}
S_{P}\left(\left\{\mu_{i}\right\}\right)= & S_{n-1,+}\left(\mu_{n}\right) S_{n-2,+}\left(\mu_{n-1}+\mu_{n}\right) \\
& \cdots S_{1,+}\left(\mu_{2}+\mu_{3}+\cdots+\mu_{n}\right) \delta\left(\sum \mu_{i}\right) .
\end{aligned}
$$

As $\sum \mu_{i}=\sum f_{i}$, we deduce that the Dirac distribution implies that the FT $S(\boldsymbol{f})$ is zero outside the stationary manifold defined after (4). The exact calculation of this FT implies the sum with respect to the permutations, as indicated in (33), and this calculation depends strongly on the structure of the function $s(\cdot)$. We shall first establish an interesting result concerning Dirac and principal value distributions used for the following discussion.

\section{Application to the Constant Signal}

In this case we have $s_{n}(\theta)=1$. As a consequence, the functions $S(\mu)$ defined by (36) are all equal and their value is the FT of the unit step function $u(t)$ equal to 0 for $t<0$ and to 1 for $t>0$. It is clear that $u(t)=(1 / 2)[1+v(t)]$, where $v(t)$ is the "sign of $t$ " function, equal to -1 for $t<0$ and to +1 for $t>0$. The FT of $v(t)$ is the principal value distribution, or

$$
V(f)=\frac{1}{\pi j} \frac{\mathrm{p} . \mathrm{v} .}{f}=\frac{\mathrm{p} \cdot \mathrm{v} .}{\pi j f}
$$

which yields the classical expression of the FT of $u(t)$

$$
U(f)=(1 / 2)[\delta(f)+V(f)]=\frac{1}{2}\left[\delta(f)+\frac{\mathrm{p} \cdot \mathrm{v} .}{\pi j f}\right] .
$$

Applying (33) and (37), where the order of the frequencies is inverted, yields the FT of 1, or

$$
\begin{array}{r}
\delta\left(f_{1}+f_{2}+\cdots+f_{n}\right) \sum_{P} U\left(\mu_{1}\right) U\left(\mu_{1}+\mu_{2}\right) \\
\cdots U\left(\mu_{1}+\mu_{2}+\cdots+\mu_{n-1}\right) \\
\left.=\delta\left(f_{1}\right) \delta f_{2}\right) \cdots \delta\left(f_{n}\right)=\delta(f)
\end{array}
$$

where, as in (33), the sum is extended to all the $n$ ! permutations of the $n$ frequencies $f_{i}$. As $U(f)$ is the sum of two distributions (Dirac and principal value), this expression establishes a relation between the Dirac and the principal value distributions that deserves additional comments.

For $n=2$ the result is obvious because $V(f)$ is an odd distribution and $\delta(f)$ an even distribution.

For $n=3$ one can write (40) in the form

$$
\delta\left(f_{1}+f_{2}+f_{3}\right) \sum_{P} U\left(\mu_{1}\right) U\left(-\mu_{2}\right)=\delta\left(f_{1}\right) \delta\left(f_{2}\right) \delta\left(f_{3}\right)
$$

where the sum is extended to the six permutations of the three frequencies $f_{i}$. Replacing $U(\cdot)$ by its value and using the symmetry properties of $\delta(\cdot)$ and $V(\cdot)$ yield that the products 
such that $\delta(\cdot) V(\cdot)$ disappear in such a way that (41) can be written in the form

$$
\begin{gathered}
\delta\left(f_{1}+f_{2}+f_{3}\right)\left[V\left(f_{1}\right) V\left(f_{2}\right)+V\left(f_{2}\right) V\left(f_{3}\right)+V\left(f_{3}\right) V\left(f_{1}\right)\right] \\
=\delta\left(f_{1}\right) \delta\left(f_{2}\right) \delta\left(f_{3}\right) .
\end{gathered}
$$

This result can be shown directly. Indeed, the FT of the left-hand side term is

$v\left(t_{1}-t_{2}\right) v\left(t_{1}-t_{3}\right)+v\left(t_{2}-t_{3}\right) v\left(t_{2}-t_{1}\right)+v\left(t_{3}-t_{1}\right) v\left(t_{3}-t_{2}\right)$

and it is obvious that this sum is equal to 1 , which implies the FT $\delta\left(f_{1}\right) \delta\left(f_{2}\right) \delta\left(f_{3}\right)$.

For arbitrary value of $n$ it is clear that the product of functions $U(\cdot)$ defined by (39) gives in the left-hand side of (40) the term $\left[n ! / 2^{n-1}\right] \delta(\boldsymbol{f})$. This implies that an appropriate sum of products of delta and principal value distribution can generate a term proportional to $\delta(\boldsymbol{f})$, and this point shall be used in the discussion that follows.

\section{CAlCulation OF POlysPeCtRA}

\section{A. Bispectrum of the Random-Jump Signal}

The third-order moment of this signal is given by (15) and applying (33) and (37) yields

$$
M_{3}(\boldsymbol{f})=m_{3} \delta\left(f_{1}+f_{2}+f_{3}\right) \sum_{P} S\left(\mu_{1}\right) S\left(-\mu_{2}\right)
$$

where $S(\mu)$ is defined by (36) in which $s_{n-1}(\theta)$ is replaced by $\exp (-\lambda \theta)$. As a result, $S(\mu)=1 /(\lambda+2 \pi j \mu)$. Calculating the sum of the six terms corresponding to the six permutations of the frequencies $f_{i}$ and using the fact that $S(-\mu)=S^{*}(\mu)$ yields the bispectrum defined by (4), or

$$
\begin{aligned}
& \Gamma_{2}\left(f_{1}, f_{2}\right) \\
& \quad=2 m_{3} \operatorname{Re}\left\{S\left(f_{1}\right) S^{*}\left(f_{2}\right)+\left[S\left(f_{1}\right)+S\left(f_{2}\right)\right] S\left(f_{1}+f_{2}\right)\right\} .
\end{aligned}
$$

This expression does not have the form (11), which means that this signal is not a linear process. It is also worth pointing out that this bispectrum is a real function, which is not in general the case. This property comes from the fact that the moment $m_{3}(\boldsymbol{t})$ is an even function because the difference $\theta_{3}-\theta_{1}$ remains invariant when changing $t_{i}$ into $-t_{i}$. It is also obvious that $\Gamma_{2}\left(f_{1}, f_{2}\right)$ remains invariant in any change of a frequency $f_{i}$ into $-\left(f_{1}+f_{2}\right)$, a property discussed in Section III-A.

\section{B. Trispectra of Ordered Signals}

We shall begin by calculating the trispectrum associated with the moment function $m_{4}\left(\left\{t_{i}\right\}\right)$ defined by (13), or equal to $\gamma\left(\theta_{2}-\theta_{1}\right) \gamma\left(\theta_{4}-\theta_{3}\right)$. The corresponding spectral moment $M_{4}\left(\left\{f_{i}\right\}\right)$ is given by (33), which is a sum of 24 terms in the form (37), or

$$
\begin{aligned}
S_{P}\left(\left\{\mu_{i}\right\}\right)= & \Gamma_{+}\left(\mu_{4}\right) U\left(\mu_{3}+\mu_{4}\right) \Gamma_{+}\left(\mu_{2}+\mu_{3}+\mu_{4}\right) \\
& \times \delta\left(\mu_{1}+\mu_{2}+\mu_{3}+\mu_{4}\right) .
\end{aligned}
$$

In this expression $\Gamma_{+}(\mu)$ is defined by

$$
\Gamma_{+}(\mu)=\int_{0}^{+\infty} \gamma(\tau) \exp (-2 \pi j \mu \tau) d \tau .
$$

Note that

$$
\Gamma_{+}(f)+\Gamma_{+}(-f)=\Gamma_{+}(f)+\Gamma_{+}^{*}(f)=\Gamma(f)
$$

where $\Gamma(f)$ is the power spectrum, FT of the correlation function $\gamma(\tau)$. Furthermore, when $\gamma(\tau)$ is defined by (14) we have seen just above that $\Gamma_{+}(f)=1 /[\lambda+2 \pi j f]$. This function is bounded and continuous. We assume that these properties are satisfied for all the functions $\Gamma_{+}(f)$ used hereafter. Physically this especially means that we exclude from our discussion the case where there are spectral lines in the spectrum of $x(t)$.

By using (39), the standard properties of the Dirac distribution and the fact that $V(\mu)$ is an odd function, one can express (45) in the form

$$
\begin{aligned}
S_{P}\left(\left\{\mu_{i}\right\}\right)= & (1 / 2) \Gamma_{+}\left(\mu_{2}\right) \Gamma_{+}\left(\mu_{4}\right) \delta\left(\mu_{1}+\mu_{2}\right) \delta\left(\mu_{3}+\mu_{4}\right) \\
& -(1 / 2) V\left(\mu_{1}+\mu_{2}\right) \Gamma_{+}\left(-\mu_{1}\right) \\
& \times \Gamma_{+}\left(-\mu_{1}-\mu_{2}-\mu_{3}\right) \delta\left(\mu_{1}+\mu_{2}+\mu_{3}+\mu_{4}\right) .
\end{aligned}
$$

It is worth presenting some comments on this result. It especially means that the FT of the function equal to $\gamma\left(t_{2}-\right.$ $\left.t_{1}\right) \gamma\left(t_{4}-t_{3}\right)$ for $t_{1}<t_{2}<t_{3}<t_{4}$ and zero otherwise, is given by (48), where the frequencies $\mu_{i}$ are replaced by $f_{i}$. This expression is a sum of two terms. The first corresponds to a distribution on the first normal manifold defined by $f_{1}+f_{2}=$ $f_{3}+f_{4}=0$ with the density $(1 / 2) \Gamma_{+}\left(f_{2}\right) \Gamma_{+}\left(f_{4}\right)$. The second corresponds to a distribution on the stationary manifold with the density $(-1 / 2) V\left(f_{1}+f_{2}\right) \Gamma_{+}\left(-f_{1}\right) \Gamma_{+}\left(f_{4}\right)$. This density makes use of the function $V(f)$ defined by (38). The fact that this function becomes infinite for $f=0$ implies that the distribution on the stationary manifold becomes infinite for the frequencies belonging to the first normal manifold for which $f_{1}+f_{2}=0$.

Returning to the calculation of $M_{4}\left(\left\{f_{i}\right\}\right)$ we have now to make the sum of 24 terms like (48) and corresponding to the domains of $t_{1} \times t_{2} \times t_{3} \times t_{4}$ defined by all the permutations of the time instants $t_{i}$. These terms are deduced from (48) by considering the 24 possible permutations of the four frequencies $f_{i}$.

Let us first consider the contribution of the normal manifolds. The first normal manifold is defined by $f_{1}+f_{2}=0$ and $f_{3}+f_{4}=0$. There are eight permutations of the $f_{i}$ 's giving a contribution of (48) to this manifold. These permutations are

$$
\begin{array}{llllllll}
f_{1} & f_{2} & f_{3} & f_{4} & f_{3} & f_{4} & f_{1} & f_{2} \\
f_{2} & f_{1} & f_{3} & f_{4} & f_{3} & f_{4} & f_{2} & f_{1} \\
f_{1} & f_{2} & f_{4} & f_{3} & f_{4} & f_{3} & f_{1} & f_{2} \\
f_{2} & f_{1} & f_{4} & f_{3} & f_{4} & f_{3} & f_{2} & f_{1} .
\end{array}
$$

The term $\Gamma_{+}\left(\mu_{2}\right) \Gamma_{+}\left(\mu_{4}\right)$ is the same for the permutations of the right-hand side and the left-hand side. This eliminates the factor $1 / 2$ appearing in (48). The sum of the four remaining terms can be written as

$$
S=\left[\Gamma_{+}\left(f_{1}\right)+\Gamma_{+}\left(f_{2}\right)\right]\left[\Gamma_{+}\left(f_{3}\right)+\Gamma_{+}\left(f_{4}\right)\right] .
$$

But on the first normal manifold $f_{2}=-f_{1}$ and $f_{4}=-f_{3}$. Using (47) yields

$$
S=\Gamma\left(f_{1}\right) \Gamma\left(f_{3}\right)
$$


which is the coefficient appearing in the first term of (6). Repeating the same procedure to the other two manifolds, we conclude that the FT of $m_{4}\left(\left\{t_{i}\right\}\right)=\gamma\left(\theta_{2}-\theta_{1}\right) \gamma\left(\theta_{4}-\theta_{3}\right)$ introduces a distribution on the normal manifolds with a normal density, as in (6). We can deduce from this result, that if we use the cumulants instead of the moments, the density on the normal manifolds becomes equal to zero.

The nonnormal property is therefore due to the contribution of the frequencies belonging to the stationary manifold but not to the normal manifolds. For this purpose we have to analyze the last term of (48). The density on this manifold is

$$
-(1 / 2) V\left(\mu_{1}+\mu_{2}\right) \Gamma_{+}\left(-\mu_{1}\right) \Gamma_{+}\left(-\mu_{1}-\mu_{2}-\mu_{3}\right) .
$$

The eight permutations of the $f_{i}$ 's appearing in (49) introduce the common term $V\left(f_{1}+f_{2}\right)$ because $V\left(f_{3}+f_{4}\right)=$ $-V\left(f_{1}+f_{2}\right)$. By grouping these eight terms we can write the contribution of the corresponding permutations to the final trispectrum in the form $V\left(f_{1}+f_{2}\right) A\left(f_{1}, f_{2}, f_{3}, f_{4}\right)$. It remains to calculate this function $A\left(f_{1}, f_{2}, f_{3}, f_{4}\right)$. This can be realized by grouping conveniently the eight terms, which gives

$$
\begin{aligned}
& A\left(f_{1}, f_{2}, f_{3}, f_{4}\right) \\
& \quad=j \operatorname{Im}\left\{\left[\Gamma_{+}\left(f_{1}\right)+\Gamma_{+}\left(f_{2}\right)\right]\left[\Gamma_{+}\left(-f_{3}\right)+\Gamma_{+}\left(-f_{4}\right)\right]\right\}
\end{aligned}
$$

in which $f_{4}=-\left(f_{1}+f_{2}+f_{3}\right)$ because of the stationarity. As a result of these calculations, the complete expression of the moment trispectrum is

$$
\Gamma_{3}\left(f_{1}, f_{2}, f_{3}\right)=T_{N}+T_{n N}
$$

where $T_{N}$ is the normal term given by (8) and $T_{n N}$ is the nonnormal term that can be expressed as

$$
\begin{aligned}
T_{n N}= & V\left(f_{1}+f_{2}\right) A\left(f_{1}, f_{2}, f_{3}, f_{4}\right) \\
& +V\left(f_{1}+f_{3}\right) A\left(f_{1}, f_{3}, f_{2}, f_{4}\right) \\
& +V\left(f_{1}+f_{4}\right) A\left(f_{1}, f_{4}, f_{2}, f_{3}\right) .
\end{aligned}
$$

This can be written in a less symmetric form by noting that $f_{4}=-\left(f_{1}+f_{2}+f_{3}\right)$.

As indicated in Section II, the cumulant trispectrum can be deduced from the moment trispectrum by subtracting the normal contribution. This implies that (54) is in fact the cumulant trispectrum $\Gamma_{c, 3}\left(f_{1}, f_{2}, f_{3}\right)$ of the signal with moments defined by (13). As boundedness is one of the important properties of cumulant trispectra, it is interesting to verify whether or not this is satisfied for this signal. Apparently it is not verified because of the presence of the terms $V\left(f_{i}+f_{j}\right)$ in (54), and these functions become infinite on the normal manifolds. However, a more detailed analysis is required because the terms $A(\cdot, \cdot, \cdot, \cdot)$ tends to zero on these manifolds. Therefore, let us study the situation when $f_{1}+f_{2}$ tends to 0 and $f_{3} \neq \pm f_{1}$. In this case the last two terms of (54) remain finite and only the first term must be analyzed. For this purpose let us write (52) in the form $A=j \operatorname{Im}[T]$, which defines $T$, and $f_{2}=-f_{1}+\epsilon$. As a result, we have

$$
T=\left[\Gamma_{+}\left(f_{1}\right)+\Gamma_{+}\left(-f_{1}+\epsilon\right)\right]\left[\Gamma_{+}\left(-f_{3}\right)+\Gamma_{+}\left(f_{3}+\epsilon\right)\right] .
$$

As $\Gamma_{+}(f)$ is continuous, it results from (47) that when $\epsilon \rightarrow 0$ this expression tends to $\Gamma\left(f_{1}\right) \Gamma\left(f_{3}\right)$ which is positive. As a consequence, the imaginary part tends to 0 at least in $\epsilon$ and as $V\left(f_{1}+f_{2}\right)=V(\epsilon)=1 /(\pi j \epsilon)$, the term $A$ remains finite. In conclusion, even if the principal value distribution is present, the cumulant trispectrum remains bounded, as for a linear process. However, it is easy to verify that this signal is not a linear process because (11) implies that $\Gamma_{c, 3}(f, 0,0)$ is proportional to $\Gamma(f)$, and this property is obviously not satisfied for the cumulant trispectrum (54).

In order to obtain the trispectrum associated with the moment given by (17) it remains to calculate the FT of the last term of this expression. For simplicity we do not take into account in the calculation the coefficient $m_{4}-m_{2}^{2}$. The FT of $\exp \left[-\lambda\left(\theta_{4}-\theta_{1}\right)\right]$ is given by (33) in which

$S_{P}\left(\left\{\mu_{i}\right\}\right)=S\left(-\mu_{1}\right) S\left(\mu_{3}+\mu_{4}\right) S\left(\mu_{4}\right) \delta\left(\mu_{1}+\mu_{2}+\mu_{3}+\mu_{4}\right)$

where $S(\mu)$ is the function used in (43). It appears immediately that this term is bounded on the stationary manifold, and therefore is the same when using cumulants instead of moments. In order to calculate its value we note that the four permutations appearing in the left table of (48) give the contribution

$$
C_{12}\left(\left\{f_{i}\right\}\right)=\left[S\left(-f_{1}\right)+S\left(-f_{2}\right)\right]\left[S\left(f_{3}\right)+S\left(f_{4}\right)\right] S\left(f_{3}+f_{4}\right) .
$$

It remains to calculate the sum of six such terms that can be written as $\left(C_{12}+C_{34}\right)\left(C_{13}+C_{24}\right)\left(C_{14}+C_{23}\right)$. As the terms in the brackets are complex conjugate, the moment trispectrum of (17) becomes

$$
\begin{aligned}
\Gamma_{3}\left(f_{1}, f_{2}, f_{3}\right)= & T_{N}+T_{n N}+2\left(m_{4}-m_{2}^{2}\right) \\
& \times \operatorname{Re}\left[C_{12}\left(\left\{f_{i}\right\}\right)+C_{13}\left(\left\{f_{i}\right\}\right)+C_{14}\left(\left\{f_{i}\right\}\right)\right]
\end{aligned}
$$

where $T_{N}$ and $T_{n N}$ are defined in (53). Note that the frequency $f_{4}$ appearing in the terms $C_{k l}\left(\left\{f_{i}\right\}\right)$ must be replaced by $-f_{1}-f_{2}-f_{3}$.

Let us now consider moment functions like (21). The FT of the first term is given by (53) and (54). The FT of the $\exp \left[-\lambda\left(\theta_{4}-\theta_{1}\right)\right]$ is proportional to the last term of (58). The FT of the second term of (21) introduces a convolution. The calculation of this convolution requires the knowledge of the structure of the term $\mu_{4}\left(\left\{t_{i}\right\}\right)$. However, whatever this structure, even if its FT introduces distributions on submanifolds of the stationary manifolds, it is easy to verify that, because of the convolution with a function bounded on this manifold, the convolution possesses also this property. In conclusion, all the cumulant trispectra of ordered signal considered here introduce a bounded cumulant trispectrum, exactly as for linear processes, even if these signals are not linear processes.

\section{Polyspectra of the Random Telegraph Signal}

1) General Structure: The previous discussion shows that the most interesting term for the trispectrum has the structure (13) which is the moment associated with the random telegraph signal and appears also as component of moments of various other ordered signals. We shall therefore consider now only 
this term. Its FT is given by (33) where $S_{P}(\cdot)$ is defined by (37). In the present case, this expression can be written in the form

$$
\begin{aligned}
S_{P}\left(\left\{\mu_{i}\right\}\right)= & \Gamma_{+}\left(\mu_{1}\right) \Gamma_{+}\left(\mu_{1}+\mu_{2}+\mu_{3}\right) \\
& \cdots \Gamma_{+}\left(\mu_{1}+\mu_{2}+\cdots+\mu_{2 n-1}\right) U\left(\mu_{1}+\mu_{2}\right) \\
& \times U\left(\mu_{1}+\mu_{2}+\mu_{3}+\mu_{4}\right) \\
& \cdots U\left(\mu_{1}+\mu_{2}+\cdots+\mu_{2 n-2}\right) \\
& \times \delta\left(\mu_{1}+\mu_{2}+\cdots+\mu_{2 n}\right)
\end{aligned}
$$

where $\Gamma_{+}(\mu)$ is defined by (46). As the function (or distribution) $U(\cdot)$ is, according to (39), the sum of the Dirac and principal value distributions, this expression exhibits a sum of terms containing products of these distributions.

The case $n=2$ previously studied is especially simple because there is only one function $U(\cdot)$ in (59) and it is obvious to separate the role of the delta and of the principal value distributions. This is no longer true for $n>2$, and, for example, it is not possible to only consider the product of delta distributions to calculate the density on the normal manifolds. Let us explain this point. It is clear that this product leads to a distribution on these manifolds. In order to calculate the corresponding density, let us consider the first normal manifold defined by (7). The permutations $\left(f_{1}, f_{2}\right)$ and $\left(f_{2}, f_{1}\right)$ associated with the same permutation of the other frequencies $f_{i}, 3 \leq f_{i} \leq 2 n$ introduce the term

$$
\Gamma_{+}\left(f_{1}\right)+\Gamma_{+}\left(-f_{1}\right)=\Gamma\left(f_{1}\right)
$$

according to (47). There are $n$ ! permutations of the pairs of frequencies such as $\left(f_{1}, f_{2}\right)$. This introduces $n$ ! terms giving the same contribution on this normal manifold. This contribution is equal to $1 / 2^{n-1}$ because of the factor $1 / 2$ existing in the $n-1$ terms $U(\cdot)$ defined by (39) and appearing in (59). Combining all these results indicates that the density on the first normal manifold coming from the product of delta functions only in (59) is

$$
D\left(\left\{f_{i}\right\}\right)=\frac{n !}{2^{n-1}} \Gamma\left(f_{1}\right) \Gamma\left(f_{3}\right) \cdots \Gamma\left(f_{2 n-1}\right) .
$$

Except for the case $n=2$ already analyzed, this density on this normal manifold is no longer normal. Furthermore, it is worth noting that the coefficient appearing in the expression of this density is the same as the one obtained when discussing (40).

In reality, the calculation of the density on the normal manifold is much more complex because this density is not only coming from the delta distribution appearing in (39) but also from an appropriate grouping of principal values distributions, according to (40). In order to avoid long algebraic expressions, we shall first consider the case $n=6$ which is still tractable and also introduces the general principles allowing the calculation of the density on the normal manifolds.

2) Spectral Moment for $n=3$ : In this case, (59) takes the form

$$
\begin{aligned}
S_{P}\left(\left\{\mu_{i}\right\}\right)= & \Gamma_{+}\left(\mu_{1}\right) \Gamma_{+}\left(\mu_{1}+\mu_{2}+\mu_{3}\right) \\
& \times \Gamma_{+}\left(\mu_{1}+\mu_{2}+\mu_{3}+\mu_{4}+\mu_{5}\right) \\
& \times U\left(\mu_{1}+\mu_{2}\right) U\left(\mu_{1}+\mu_{2}+\mu_{3}+\mu_{4}\right) \\
& \times \delta\left(\mu_{1}+\mu_{2}+\mu_{3}+\mu_{4}+\mu_{5}+\mu_{6}\right) .
\end{aligned}
$$

The spectral moment function is given by (33) in which the sum contains $6 !=720$ permutations of the frequencies $f_{i}$. The last term of (61) shows that, as expected, the spectral moment $S(\boldsymbol{f})$ is zero outside the stationary manifold. As the distribution $U(\mu)$ appearing in (61) is singular for $\mu=0$, it appears three different situations.

a) Normal Manifolds. They are defined by the fact that there are three and only three equations of the type $f_{i}+f_{j}=0$. As an example, the first normal manifold is defined by

$$
f_{1}+f_{2}=0 \quad f_{3}+f_{4}=0 \quad f_{5}+f_{6}=0 .
$$

There are 15 such normal manifolds and it is clear that $2^{3} \cdot 3 !=48$ permutations of the frequencies $f_{i}$ leave (62) invariant. It is the same for the other normal manifolds, which gives $15 \times 48=720$ permutations, as indicated above.

b) Manifolds 2-4. They are submanifolds of the stationary manifolds defined by one and only one equation on the type $f_{i}+f_{j}=0$ between two specific frequencies $f_{i}$ and $f_{j}$. For example, the first manifold of this type is

$$
f_{1}+f_{2}=0 \quad f_{3}+f_{4}+f_{5}+f_{6}=0 .
$$

There are clearly 15 such manifolds and $2 \times 4$ ! permutations of the frequencies $f_{i}$ leave (63) invariant. This again gives the number of 720 permutations of the six frequencies $f_{i}$.

c) Stationary Manifold. There is no coupling such $f_{i}+$ $f_{j}=0$ between frequencies and therefore the product of distributions $U(\cdot)$ in (61) remains bounded. It is worth pointing out that, because of the fact that these distributions contain an even number of frequencies $f_{i}$, the submanifolds such that $f_{1}+f_{2}+f_{3}=0$ do not appear in $S(\boldsymbol{f})$.

3) Density on the Normal Manifolds: Let $\nu_{i}$ be a set of six frequencies defining a point on the first normal manifold. This means that these frequencies satisfy (62) where each $f_{i}$ is replaced by $\nu_{i}$. Let $D_{1}$ be the domain of frequencies $f_{i}$ defined by

$$
f_{i}=\nu_{i}+\epsilon_{i}, \quad\left|\epsilon_{i}\right|<b / 2 .
$$

The index 1 in $D_{1}$ means that this domain is a neighborhood of the first normal manifold. The value of the density on this manifold is obtained by studying the limit when $b \rightarrow 0$ of the integral

$$
I\left(D_{1}\right)=\int_{D_{1}} S(\boldsymbol{f}) d \boldsymbol{f} .
$$

Let $\mathcal{P}_{1}$ be the 48 permutations of the frequencies $f_{i}$ leaving (62) invariant and $\mathcal{P}_{1}^{C}$ the $720-48=672$ other permutations of the frequencies $f_{i}$. Using (33) in (65) yields $I\left(D_{1}\right)=$ $I\left(D_{1}, \mathcal{P}_{1}\right)+I\left(D_{1}, \mathcal{P}_{1}^{C}\right)$ with

$$
I\left(D_{1}, \mathcal{P}\right)=\int_{D_{1}} \sum_{\mathcal{P}} S_{P}\left(\left\{f_{i}\right\}\right) d f .
$$

Consider first the integral $I\left(D_{1}, \mathcal{P}_{1}^{C}\right)$. Let $\nu_{i}^{\prime}$ be the six frequencies deduced from the $\nu_{i}$ 's by an arbitrary permutation 
belonging to $\mathcal{P}_{1}^{C}$. As the $\nu_{i}$ 's correspond to a point on the first normal manifold, we have $\nu_{1}^{\prime}+\nu_{2}^{\prime} \neq 0$ and $\nu_{3}^{\prime}+$ $\nu_{4}^{\prime} \neq 0$. Therefore, for $b$ sufficiently small, the frequencies $\mu_{j}$ corresponding to any permutation of the frequencies $f_{i}$ belonging to $\mathcal{P}_{1}^{C}$ cannot satisfy $\mu_{1}+\mu_{2}=0, \mu_{1}+\mu_{2}+$ $\mu_{3}+\mu_{4}=0$. As a consequence, the terms delta coming from the distributions $U(\cdot)$ of (61) disappears. Furthermore, the terms $V(\cdot)$ are bounded because the only singularity of $V(f)$ defined by (38) is for $f=0$. Finally, as the function $\Gamma_{+}(\mu)$ is bounded and because of the last term $\delta(\cdot)$ in (61), we have $\left|I\left(D_{1}, \mathcal{P}_{1}^{C}\right)\right|<B b^{\tilde{5}}$.

Consider now the integral $I\left(D_{1}, \mathcal{P}_{1}\right)$. The first permutation of $\mathcal{P}_{1}$ is the identical permutation for which we have $\mu_{i}=f_{i}$. Using (64) and the point that the frequencies $\nu_{i}$ belong to the first normal manifold yields

$$
\begin{aligned}
\mu_{1} & =\nu_{1}+\epsilon_{1} \\
\mu_{1}+\mu_{2}+\mu_{3} & =\nu_{3}+\epsilon_{1}+\epsilon_{2}+\epsilon_{3} \\
\mu_{1}+\cdots+\mu_{5} & =\nu_{5}+\epsilon_{1}+\cdots+\epsilon_{5}
\end{aligned}
$$

As a consequence, the term (61) corresponding to the identical permutation $P_{1}$ can be written as

$$
\begin{aligned}
S_{P_{1}}\left(\left\{\mu_{i}\right\}\right)= & {\left[\Gamma_{+}\left(\nu_{1}\right) \Gamma_{+}\left(\nu_{3}\right) \Gamma_{+}\left(\nu_{5}\right)+K_{1}\left(\left\{\epsilon_{i}\right\}\right)\right] } \\
& \times U\left(\epsilon_{1}+\epsilon_{2}\right) U\left(\epsilon_{1}+\cdots+\epsilon_{4}\right) \delta\left(\epsilon_{1}+\cdots+\epsilon_{6}\right)
\end{aligned}
$$

and $\left.\mid K_{1}\left(\left\{\epsilon_{i}\right\}\right)\right] \mid<k_{1} b^{3}$ because of the assumptions on the functions $\Gamma_{+}(\mu)$. Permuting $f_{1}$ and $f_{2}$ while leaving unchanged the other frequencies yields to replace in this expression $\Gamma_{+}\left(\nu_{1}\right)$ by $\Gamma_{+}\left(\nu_{2}\right)$. But as $\nu_{2}=-\nu_{1}$, we deduce from (47) that the sum corresponding to these two permutations introduces the term $\Gamma\left(\nu_{1}\right)$. Making the same operation for the other frequencies of (68) yields that the sum of the functions $S_{P_{1}}\left(\left\{\mu_{i}\right\}\right)$ extended to the permutations of $\mathcal{P}_{1}$ can be expressed as a product $A B$. The term $A$ corresponds to the $2^{3}$ permutations of $\mathcal{P}_{1}$ between $f_{1}$ and $f_{2}, f_{3}$ and $f_{4}$, and $f_{5}$ and $f_{6}$. Its value is

$$
\left.A=\Gamma\left(\nu_{1}\right) \Gamma\left(\nu_{3}\right) \Gamma\left(\nu_{\tilde{5}}\right)+K\left(\left\{\epsilon_{i}\right\}\right), \mid K\left(\left\{\epsilon_{i}\right\}\right)\right] \mid<k b^{3} .
$$

The term $B$ corresponds to the other permutations of $\mathcal{P}_{1}$. By introducing $\lambda_{1}=\epsilon_{1}+\epsilon_{2}, \lambda_{2}=\epsilon_{3}+\epsilon_{4}$, and $\lambda_{3}=\epsilon_{5}+\epsilon_{6}$ we can write

$$
B=\sum_{P_{1}^{\prime}} U\left(\lambda_{1}\right) U\left(\lambda_{1}+\lambda_{2}\right) \delta\left(\lambda_{1}+\lambda_{2}+\lambda_{3}\right)
$$

where $P_{1}^{\prime}$ means the six permutations of the threes numbers $\lambda_{i}$. From (40) we deduce that

$$
B=\delta\left(\epsilon_{1}+\epsilon_{2}\right) \delta\left(\epsilon_{3}+\epsilon_{4}\right) \delta\left(\epsilon_{5}+\epsilon_{6}\right) .
$$

Introducing this expression in (66) yields

$$
\begin{aligned}
I\left(D_{1}, \mathcal{P}_{1}\right)= & \int_{D_{1}}\left[\Gamma\left(\nu_{1}\right) \Gamma\left(\nu_{3}\right) \Gamma\left(\nu_{5}\right)+K\left(\left\{\epsilon_{i}\right\}\right)\right] \\
& \times \delta\left(\epsilon_{1}+\epsilon_{2}\right) \delta\left(\epsilon_{3}+\epsilon_{4}\right) \delta\left(\epsilon_{5}+\epsilon_{6}\right) d \epsilon_{1} \cdots d \epsilon_{6} .
\end{aligned}
$$

This can easily be written as

$$
I\left(D_{1}, \mathcal{P}_{1}\right)=\Gamma\left(\nu_{1}\right) \Gamma\left(\nu_{3}\right) \Gamma\left(\nu_{5}\right) b^{3}+T
$$

and the previous inequalities give $|T|<b^{6}$. In conclusion, (65) can be expressed as

$$
I\left(D_{1}, \mathcal{P}_{1}\right)=b^{3}\left[\Gamma\left(\nu_{1}\right) \Gamma\left(\nu_{3}\right) \Gamma\left(\nu_{5}\right)+c b^{2}\right]
$$

which shows that the density on the first normal manifold is $\Gamma\left(\nu_{1}\right) \Gamma\left(\nu_{3}\right) \Gamma\left(\nu_{5}\right)$. It is a normal density, as defined after $(7)$.

It is obvious that the same reasoning can be presented for the 14 other manifolds, and the conclusion is that the spectral moment function $M_{6}\left(\left\{f_{i}\right\}\right)$ of the RTS introduces a normal density on the normal manifolds.

As the cornerstone of the reasoning is (40) valid for any $n$, the result can be extended for any spectral moment $M_{2 n}\left(\left\{f_{i}\right\}\right)$. This especially means that the nonnormal property of the RTS comes from the contributions of nonzero density outside the normal manifolds.

4) Density on Nonnormal Manifolds: Suppose as previously that $n=3$. The spectral moment function is given by the sum with respect to the 720 permutations of terms like (61).

Consider the 48 permutations such that the sets of frequencies $\left(f_{1}, f_{2}, f_{3}, f_{4}\right)$ and $\left(f_{5}, f_{6}\right)$ remain invariant. Suppose also that there is no coupling such that $f_{i}+f_{j}=0$ between frequencies $f_{i}, 1 \leq i \leq 4$. We can write (61) in the form

$$
\begin{aligned}
S_{P}\left(\left\{\mu_{i}\right\}\right)= & \Gamma_{+}\left(\mu_{1}\right) U\left(\mu_{1}+\mu_{2}\right) \Gamma_{+}\left(\mu_{1}+\mu_{2}+\mu_{3}\right) \\
& \times \Gamma_{+}\left(-\mu_{6}\right) U\left(-\mu_{5}-\mu_{6}\right) \delta\left(\sum \mu_{i}\right) .
\end{aligned}
$$

Our assumption of no coupling of frequencies yields

$$
U\left(\mu_{1}+\mu_{2}\right)=(1 / 2) V\left(\mu_{1}+\mu_{2}\right) \text {. }
$$

As $U(\cdot)$ is given by (39) we consider separately the contributions of the two terms.

a) Terms in Delta: The corresponding contribution to (73) can be written as

$$
\begin{aligned}
S_{P, \delta}\left(\left\{\mu_{i}\right\}\right)= & (1 / 2) \Gamma_{+}\left(\mu_{1}\right) V\left(\mu_{1}+\mu_{2}\right) \Gamma_{+}\left(\mu_{1}+\mu_{2}+\mu_{3}\right) \\
& \times \delta\left(\mu_{1}+\cdots+\mu_{4}\right) \times(1 / 2) \Gamma_{+}\left(-\mu_{6}\right) \\
& \times \delta\left(\mu_{5}+\mu_{6}\right) .
\end{aligned}
$$

The sum with respect to the 24 permutations of the frequencies $f_{i}, 1 \leq i \leq 4$, yields the term $T_{n N}$ given by (54). The sum with respect to the two permutations of the frequencies $f_{5}$ and $f_{6}$ yields $(1 / 2) \Gamma\left(\mu_{5}\right)$. This gives the density on the manifold defined by

$$
f_{1}+f_{2}+f_{3}+f_{4}=0 \quad f_{5}+f_{6}=0 .
$$

It is obvious that the same reasoning can be presented for every manifold of the type 2-4 introduced in Section V-C2). 
b) Terms $V(\cdot)$ : This corresponds to the value of the density in the neighborhood of the manifold defined by (75). For this consider six frequencies $\nu_{i}$ satisfying (75) and the frequencies $f_{i}$ defined by (64). The corresponding contribution to (73) is now

$$
\begin{aligned}
S_{P, V}\left(\left\{\mu_{i}\right\}\right)= & (1 / 2) \Gamma_{+}\left(\nu_{1}+\epsilon_{1}\right) V\left(\nu_{1}+\nu_{2}+\epsilon_{1}+\epsilon_{2}\right) \\
& \times \Gamma_{+}\left(-\nu_{4}+\epsilon_{1}+\epsilon_{2}+\epsilon_{3}\right) \\
& \times \Gamma_{+}\left(-\nu_{6}-\epsilon_{6}\right) V\left(\epsilon_{\check{2}}+\epsilon_{6}\right) .
\end{aligned}
$$

The product of the first three terms in bounded and its sum tends to $T_{n N}$ when the $\epsilon_{i}$ 's tend to 0 . Because of the term $V\left(\epsilon_{5}+\epsilon_{6}\right)$ which tends to infinity when approaching the stationary manifold (75), it appears that the density on the stationary manifold is not bounded, as for linear processes previously discussed. All this discussion shows that there is a strong difference between the cases $n=2$ and $n>2$. In fact, it is clear that the reasoning presented for $n=3$ can be extended with only writing complexity for greater values of $n$.

\section{Trispectrum of the RTS with Compound Poisson Processes}

As indicated in Section III-D, a compound Poisson process is a Poisson process with a random density. This means that in all the previous calculations of moments it is necessary to take an expectation with respect to the positive random variable $\lambda$.

Let us analyze the changes in the results introduced by this randomness in the structure of spectral moments of an RTS. For a given value of $\lambda$ the correlation function of the signal is $\exp \left[-2 \lambda\left|t_{2}-t_{1}\right|\right]$, and the corresponding power spectrum is $\Gamma(f)=\lambda\left[\lambda^{2}+\pi^{2} f^{2}\right]^{-1}$. As $\lambda$ is now random, the power spectrum of the RTS generated by a compound Poisson process is, therefore,

$$
\Gamma(f)=\int_{0}^{\infty} p(\lambda) \frac{\lambda}{\lambda^{2}+\pi^{2} f^{2}} d \lambda
$$

where $p(\lambda)$ is the probability density function of the random density $\lambda$ of the Poisson process. These second-order statistics have been considered in [14] for the very specific case where $p(\lambda)$ is an exponential distribution. Averaging the correlation function $\exp \left[-2 \lambda\left|t_{2}-t_{1}\right|\right]$ with respect to $\lambda$ yields the correlation function $\left[1+2 a\left|t_{2}-t_{1}\right|\right]^{-1}$, where $a$ characterizes the exponential distribution.

All the calculations made to obtain the moment trispectrum are valid for each value of $\lambda$. It suffices at the end to take an expectation with respect to the random variable $\lambda$. This is especially valid for the quantity $S$ defined by (51) and giving the density on the first normal manifold. After the expectation, this density becomes

$$
S^{\prime}=\int_{0}^{\infty} p(\lambda) \frac{\lambda}{\lambda^{2}+\pi^{2} f_{1}^{2}} \frac{\lambda}{\lambda^{2}+\pi^{2} f_{3}^{2}} d \lambda .
$$

Comparing with (77) shows that this term can no longer be expressed as $\Gamma\left(f_{1}\right) \Gamma\left(f_{3}\right)$. The conclusion is then that the density on the normal manifold is no longer normal, or is no longer a product of spectral densities. This implies in particular that the cumulant trispectrum is no longer equal to zero on the normal manifolds.

\section{ASYMPTOTIC NORMALITY}

It is often admitted that passing a nonnormal signal in very narrowband filters generates a normal output. This fact is intuitively motivated by the use of the central limit theorem (see [5, p. 134]). Indeed, a narrowband filter has a slowly decreasing impulse response, and the convolution required for the calculation of the output creates a mixture of a great number of almost independent contributions, which justifies the use of the central limit theorem.

We shall see that a better understanding of this phenomenon can be obtained by reasoning in the frequency domain, and especially by using moment spectral functions. In order to simplify the discussion we shall first use the trispectrum corresponding to moments of order 4 , the extension to orders higher than 4 being discussed at the end.

\section{A. Relations Between Time and Frequency Domains}

Starting from the trispectrum given by (4), it is possible to calculate the fourth-order moment (1) by the relation

$$
\begin{aligned}
m_{4}\left(\left\{t_{i}\right\}\right)= & \iiint \Gamma_{3}\left(f_{1}, f_{2}, f_{3}\right) \exp \left\{2 \pi j \left[f_{1}\left(t_{1}-t_{4}\right)\right.\right. \\
& \left.\left.+f_{2}\left(t_{2}-t_{4}\right)+f_{3}\left(t_{3}-t_{4}\right)\right]\right\} d f_{1} d f_{2} d f_{3} .
\end{aligned}
$$

As $\Gamma_{3}$ can be decomposed as a sum of terms coming from the normal and from the stationary manifolds, we shall examine their respective contribution to the moment $m_{4}$. Suppose that the density on the first normal manifold is $A\left(f_{1}, f_{3}\right)$, which means that the first term of the right-hand side of (8) is $A\left(f_{2}, f_{3}\right) \delta\left(f_{1}+f_{2}\right)$. Inserted in (79), this first normal manifold yields the term

$$
\begin{aligned}
n_{1}\left(\left\{t_{i}\right\}\right)= & \iint A\left(f_{1}, f_{3}\right) \\
& \times \exp \left\{2 \pi j\left[f_{1}\left(t_{2}-t_{1}\right)+f_{3}\left(t_{4}-t_{3}\right)\right]\right\} d f_{1} d f_{3} .
\end{aligned}
$$

If $A\left(f_{1}, f_{3}\right)$ is given by (51), or if the density is normal, then $n_{1}\left(\left\{t_{i}\right\}\right)=\gamma\left(t_{2}-t_{1}\right) \gamma\left(t_{4}-t_{3}\right)$, which is the first term of (3), because $\gamma(\cdot)$ is a symmetric function.

\section{B. Narrowband Filtering}

In order to simplify the mathematical development, we shall use the perfect narrowband filter in what follows. However, the result obtained below can be easily extended to other cases of narrowband filters. Consider a set of filters $F_{b}$ depending on the parameter $b$ and defined by their frequency responses $G_{b}(f)$ equal to $1 / \sqrt{b}$ for $f_{0}-(b / 4)<|f|<f_{0}+(b / 4)$, and zero otherwise. This filter rejects all the frequencies, except those belonging to the bands $\left(-f_{0}-b / 4,-f_{0}+b / 4\right)$ and $\left(f_{0}-b / 4, f_{0}+b / 4\right)$. Let $y_{b}(t)$ be the output of this filter when the input is $x(t)$. The power of $y_{b}(t)$ is

$$
E\left[y_{b}^{2}(t)\right]=\int\left|G_{b}(f)\right|^{2} \Gamma(f) d f
$$

where $\Gamma(f)$ is the power spectrum of $x(t)$. Assuming that $\Gamma\left(f_{0}\right) \neq 0$, and that $\Gamma(f)$ is continuous in the neighborhood of $f_{0}$, we deduce that $E\left[y_{b}^{2}(t)\right] \simeq \Gamma\left(f_{0}\right)$, and the power of 
$y_{b}(t)$ remains constant when $b \rightarrow 0$. In other words, the term $1 / \sqrt{b}$ is a normalization constant that always appears in the statement of the central limit theorem.

In order to calculate the fourth-order moment of $y_{b}(t)$, we can use (79) in which $\Gamma_{3}$ is replaced by

$$
\begin{aligned}
& \Gamma_{3, y}\left(f_{1}, f_{2}, f_{3}\right) \\
& \quad=G_{b}\left(f_{1}\right) G_{b}\left(f_{2}\right) G_{b}\left(f_{3}\right) G_{b}^{*}\left(f_{1}+f_{2}+f_{3}\right) \Gamma_{3, x}\left(f_{1}, f_{2}, f_{3}\right)
\end{aligned}
$$

where $\Gamma_{3, x}$ is the trispectrum of $x(t)$. This expression has the same structure as (11) and is a direct consequence of the definition of the trispectrum.

\section{Contributions of the Normal Manifolds}

If the density on the first normal manifold is $A\left(f_{1}, f_{3}\right)$, its contribution to the first-order moment of $y_{b}(t)$ is

$$
\begin{aligned}
m_{y, b, 1}\left(\left\{t_{i}\right\}\right)= & \iint A\left(f_{1}, f_{3}\right)\left|G_{b}\left(f_{1}\right)\right|^{2}\left|G_{b}\left(f_{3}\right)\right|^{2} \\
& \times \exp \left\{2 \pi j\left[f_{1}\left(t_{2}-t_{1}\right)+f_{3}\left(t_{4}-t_{3}\right)\right]\right\} d f_{1} d f_{3} .
\end{aligned}
$$

It results from the definition of $G_{b}(f)$ that, as for (83), this term tends towards a finite limit when $b \rightarrow 0$. In particular, if the density is normal, which means that $A\left(f_{1}, f_{3}\right)=$ $\Gamma\left(f_{1}\right) \Gamma\left(f_{3}\right), m_{y, b, 1}$ tends to $\gamma_{y}\left(t_{2}-t_{1}\right) \gamma_{y}\left(t_{4}-t_{3}\right)$. By similar reasoning for the other two manifolds, we conclude that, when $b \rightarrow 0$, the contribution of the normal manifold is a normal moment such as (3) if and only if the density on the normal manifold is normal.

\section{Contributions of the Stationary Manifolds}

We shall now see that when $b \rightarrow 0$ this contribution tends toward zero, which physically means that narrowband spectral filtering eliminates all the contributions of the trispectrum that are not in the normal manifolds.

Suppose that the density on the stationary manifold is bounded and it was the case for all the spectral moment functions introduced above. The corresponding contribution to the fourth-order moment $m_{4}\left(\left\{t_{i}\right\}\right)$ is given by (79) where $\Gamma_{3}$ is replaced by $\Gamma_{3 y}$ of (82) in which $\Gamma_{3 x}$ is a bounded function satisfying $\left|\Gamma_{3 x}\left(f_{1}, f_{2}, f_{3}\right)\right|<M$. Because of the four functions $G_{b}(f)$ appearing in (82), the integral of (79) is bounded by $M / b^{2}$. However, the range of integration of each integral of (79) is of the order of $b$, because of the band of frequency limitations. This shows effectively that the contribution of a bounded density on the stationary manifold gives to $m_{4}$ a contribution of the order of $b$, and therefore tends towards zero when $b \rightarrow 0$.

\section{E. Generalization}

The conclusion of previous reasoning is that a conveniently normalized narrowband filtering eliminates in the trispectrum all the contributions, except those coming from the normal manifolds. Therefore, if the starting signal exhibits normal density on the normal manifolds, the fourth-order moment tends to have a structure coming from the normal distribution. This is, for example, the case with the RTS. On the other hand, if this density is not normal, and this appears, for example, with the RTS of a compound Poisson process, then there is no tendency to arrive at a normal structure.

It is worth pointing out that the same reasoning can be made in terms of cumulants and it consists in showing that all the cumulants functions tend to 0 except for $n=2$, which characterizes a normal signal.

Finally, it is clear that the reasoning is not only valid for the trispectrum but can be extended to any polyspectrum. However, it is no longer possible to admit that spectral moment functions are bounded on the stationary manifold, as seen after (76). However, the result remains the same for the following reason. Consider an integral such as

$$
I=\int_{f_{0}-\epsilon}^{f_{0}+\epsilon} V(f) h(f) d f
$$

where $h(f)$ is a bounded and continuous function. If $f_{0} \neq 0$ and if $\epsilon$ is sufficiently small, in such a way that $f_{0}-\epsilon$ and $f_{0}+\epsilon$ have the same sign, $V(f)$ is bounded and we return to the previous reasoning. This means that when $\epsilon \rightarrow 0$ the integral $I$ tends towards zero too and its value is of the order of $\epsilon$. On the other hand, if $f_{0}=0$ we can, for $h(f)$ sufficiently small, replace $h(f)$ in (84) by $h(0)$, and the integral is zero as a consequence of the definition of the principal value, or because $V(f)$ is an odd function. Therefore, the general result remains the same.

This can especially be applied to the RTS and the conclusion of this whole discussion is that this essentially nonnormal signal tends to become normal after a linear filtering with a sufficiently small bandwidth. This phenomenon has already be analyzed by considering the probability density function at the output of some filters at a given time instant [15], [16]. This density function tends effectively to that of a normal random variable, but the result is obtained only at one time instant and does not show that the output signal is normal.

\section{TIME REVERSIBILITY}

A random signal $x(t)$ is said to be time-reversible if its statistical properties are invariant when changing $t$ into $-t$. In terms of moments used in this paper, this means that $m_{n}\left(\left\{t_{i}\right\}\right)$ given by (1) is equal to $m_{n}\left(\left\{-t_{i}\right\}\right)$, for any value of $t_{i}$ 's or that $m_{n}(\boldsymbol{t})$ is an even function. Transposed into terms of spectral moments, time reversibility is characterized by $M_{n}\left(\left\{f_{i}\right\}\right)=M_{n}\left(\left\{-f_{i}\right\}\right)$ and (4) indicates that this can be written in terms of a polyspectra by the same relation. The simplest example of a time-reversible signal is that of a discrete-time, strictly white noise: it is a set of i.i.d. random variables, and we have indicated that the cumulant polyspectra are constant. Similarly, (2) or (5) show that a normal signal is time-reversible. Time reversibility is not at all a property in contradiction with ordering property. For example, it results directly from (13) that the RTS is time-reversible.

However, the important point to know is whether or not time reversibility is preserved in linear filtering and this question was stated in various ways in [17]-[19]. 
Let $x(t)$ be a time-reversible signal and $y(t)$ the signal obtained by filtering $x(t)$ in a linear filter of frequency response $G(f)$. Let us consider, for simplicity, the fourth-order spectral moment functions, the extension to any order being straightforward. These functions of $x$ and $y$ are related by

$$
M_{4 y}\left(\left\{f_{i}\right\}\right)=G\left(\left\{f_{i}\right\}\right) M_{4 x}\left(\left\{f_{i}\right\}\right)
$$

where $G\left(\left\{f_{i}\right\}\right)=G\left(f_{1}\right) G\left(f_{2}\right) G\left(f_{3}\right) G\left(f_{4}\right)$. Suppose that $x(t)$ is time-reversible. Our aim is to find conditions on $M_{4 x}\left(\left\{f_{i}\right\}\right)$ such that this property of reversibility remains valid for $y(t)$, for any filter $G(f)$. As we are working with real filters, we have $G(-f)=G^{*}(f)$. Therefore, in order to preserve reversibility, the product $G\left(f_{1}\right) G\left(f_{2}\right) G\left(f_{3}\right) G\left(f_{4}\right)$ must be real for the frequencies $f_{i}$ where $M_{4 x}\left(\left\{f_{i}\right\}\right)$ is not zero, whatever the function $G(f)$. It is clear that this implies that $M_{4 x}\left(\left\{f_{i}\right\}\right)$ is nonzero only on the normal manifolds for which the product becomes $\left|G\left(f_{i}\right)\right|^{2}\left|G\left(f_{j}\right)\right|^{2}$. However, this does not imply that $x(t)$ is normal, because nothing is required concerning the density on the normal manifolds. For example, spherically invariant stochastic processes (see [5, p. 299]) have spectral moments equal to zero outside the normal manifolds, but also a nonnormal density on these manifolds. The results reported in [18] and [19] are less restrictive. In fact, they also assume that $x(t)$ is a discrete-time white noise, or a sequence of i.i.d. random variables. This independence property implies a normal density on the normal manifold, and that is why they conclude that preservation of time reversibility requires normal distribution.

Finally note that as narrowband filtering of ordered signals leads to normality, it also introduces asymptotic time reversibility.

\section{CONCLUSION}

The study of very common signals, such as the RTS, introduces the property of time ordering. This property means that the calculation of fourth-order moments, and more generally moments of an order higher than two, requires a permutation such that the time instants are put in an increasing order. This ordering property is a consequence of the fact that time is oriented and appears in many examples of signals representing physical phenomena in real time. The HOS properties of many ordered signals are very simple to express in the time domain and various examples of higher order moment functions have been presented. On the other hand, the ordering property is not at all adapted to Fourier transformation leading to polyspectra. Indeed, in this transformation time is only a variable of integration and orientation of time does not play any role.

We presented a general method in order to calculate the polyspectra of ordered signals. The principle of this method is to decompose the domain of integration with respect to time in an appropriate way taking into account the ordering property of the signal. Furthermore, the grouping of various terms coming from this decomposition is facilitated by using some nonstandard properties of distributions. This method was especially used for some specific signals such as the RTS or various signals deduced from Poisson processes. A closed form of their polyspectra has been obtained and there are very few nonlinear models for which this is possible.

One of the most important points to study is the structure of the polyspectra on the normal manifold. If the polyspectra on these manifolds have a normal density, there are simple relationships between cumulant and moment polyspectra. Furthermore this property allows one to determine whether or not a nonnormal signal can become normal after narrowband filtering, which is a spectral approach of the central limit theorem.

Even if the ordered signals presented here are not normal, the calculation of polyspectra shows that they present a normal density on the normal manifolds and another contribution on the stationary manifold that was explicitly calculated.

\section{REFERENCES}

[1] D. Brillinger, "An introduction to polyspectra," Ann. Math. Stat., vol. 36, pp. 1351-1374, 1965.

[2] V. Chandran and S. Elgar, "A general procedure for the derivation of principal domains of higher-order spectra," IEEE Trans. Signal Processing, vol. 42, pp. 229-233, 1994

[3] M. V. Hinich and H. Messer, "On the principal domain of the discrete bispectrum," IEEE Trans. Signal Processing, vol. 43, pp. 2130-2134, 1995.

[4] J. W. Dalle Molle and M. V. Hinich, "Trispectral analysis of stationary random times series," J. Acoust. Soc. Amer., vol. 97, pp. 2963-2978, 1995.

[5] B. Picinbono, Random Signals and Systems. Englewood Cliffs, NJ: Prentice-Hall, 1993.

[6] D. Brillinger, Time Series Analysis, Data Analysis and Theory. New York: Holt, Rinehart and Winston, 1975.

[7] R. F. Dwyer, "Fourth-order spectra of Gaussian amplitude-modulated sinusoids," J. Acoust. Soc. Amer., vol. 90, pp. 918-926, 1991.

[8] P. Bondon, M. Benidir, and B. Picinbono, "Polyspectrum modeling using linear or quadratic filters," IEEE Trans. Signal Processing, vol. 41, pp. 692-702, 1993.

[9] S. Nam and E. Powers, "Application of higher order spectral analysis to cubically nonlinear system identification," IEEE Trans. Signal Processing, vol. 42, pp. 1746-1765, 1994.

[10] V. Leonov and A. Shiryaev, "On a method of calculation of semiinvariants," Theory Probab. Applic., vol. 5, pp. 460-464, 1960.

[11] B. Picinbono, "Ergodicity and fourth-order spectral moments," IEEE Trans. Inform. Theory, vol. 43, pp. 1273-1276, 1997.

[12] B. Picinbono and E. Boileau, "Coherence properties of optical fields-I: Higher order coherence functions and phase fluctuations," J. Opt. Soc. Amer., vol. 58, pp. 784-789, 1968.

[13] B. Picinbono, "On circularity," IEEE Trans. Signal Processing, vol. 42, pp. 3473-3482, 1994.

[14] R. Barakat, "Another random telegraph signal," Proc. IEEE, vol. 66, pp. $97-98,1978$.

[15] W. Wonham and A. Fuller, "Probability densities of the smoothed random telegraph signal," J. Elec. Contr., vol. 4, pp. 567-576, 1958.

[16] J. Mac Fadden, "The probability density of the output of a filter when the input is a random telegraphic signal: Differential equations method," IEEE Trans. Circuit Theory, vol. CT-6, pp. 225-233, 1959.

[17] A. Blanc-Lapierre, "Les fonctions aléatoires réversibles," C. R. l'Acad. Scien., vol. 251, pp. 1957-1959, 1960.

[18] G. Weiss, "Time reversibility of linear stochastic processes," J. Appl. Probab., vol. 12, pp. 831-836, 1975.

[19] M. Halin, C. Lefèvre, and M. Puri, "On time reversibility and the uniqueness of MA representation for non-Gaussian stationary time series," Biometrika, vol. 75, pp. 170-171, 1988. 